\title{
La forêt domaniale de Tronçais (Allier) : de l'acquisition LiDAR à l'approche archéologique de terrain
}

\author{
The Tronçais National Forest (Allier): from the LiDAR acquisition to the \\ archaeological field approach
}

\author{
Laure Laüt $^{1}$ \\ ${ }^{1}$ Maître de conférences à Paris 1 Panthéon-Sorbonne, Membre de I'UMR 8546 AOROC (ENS/PSL/EPHE/CNRS), \\ Laure.laut-taccoen@univ-paris1.fr
}

RÉSUMÉ. La forêt domaniale de Tronçais est une des plus belles chênaies d'Europe, couvrant 11600 hectares au centre de la France, dans l'Allier. Connus depuis longtemps pour abriter des vestiges, notamment gallo-romains, ce massif forestier et ses alentours ont bénéficié d'une acquisition LidarLiDAR en 2016. De très nombreux reliefs anthropiques ont alors été observés, qui complètent assez largement notre vision des occupations anciennes dans ce secteur. Un programme de prospection diachronique a été mis en place en 2017-2019, afin de vérifier sur le terrain ces indices, de mieux caractériser les structures et de les dater. Dans cet article, sont détaillées les procédures de ces interventions au sol, combinant prospection à vue, récolte de mobilier en surface et utilisation d'un détecteur de métaux. C'est aussi l'occasion de dresser un premier bilan des apports et limites de l'exploitation archéologique des données LidarLiDAR dans cette zone forestière. Ainsi, peut-on distinguer les traces que l'on voit beaucoup mieux grâce au laser scanneur aéroporté (constructions, enclos, tertres, zones d'extraction, mares, charbonnières), celles qui sont entièrement apparues (réseaux parcellaires), mais aussi celles que l'on ne voit toujours pas (occupations préhistoriques, structures en matériaux périssables) et qui échappent encore largement à notre connaissance.

ABSTRACT. The national forest of Tronçais is one of the most beautiful oak forests in Europe, covering 11,600 hectares in the center of France, in Allier. Known for a long time to shelter remains, especially Gallo-Roman, this forest and its surroundings have benefited from a LidarLiDAR acquisition in 2016. Very many anthropic reliefs were then observed, which largely complement our vision of ancient occupations in this area. A diachronic surveying program was set up in 2017-2019, in order to check on the field these clues, to characterize better the structures and to date them. In this paper, the procedures of these ground interventions, combining visual survey, collecting of artifacts on the ground and use of a metal detector, are detailed. It is also an opportunity to make an initial assessment of the assets and limits of the archaeological exploitation of LidarLiDAR data in this forest area. Thus, we can distinguish the remains that we see much better thanks to ALS (buildings, enclosures, mounds, quarries, ponds, coal platforms), those that have appeared entirely (field systems), but also those that we do not see yet (prehistoric occupations, structures in perishable materials) and which are still largely lacking to our knowledge.

MOTS-CLÉS. LiDAR, prospection, forêt, diachronie, Antiquité, parcellaire, voie, habitat, carrière, charbonnière.

KEYWORDS. LiDAR, survey, forest, diachrony, Antiquity, field system, road, settlement, quarry, coal platform.

La forêt domaniale de Tronçais et ses abords ont bénéficié d'une acquisition LiDAR en 2016 et d'un programme de prospection en 2017-2019 pour exploiter archéologiquement les indices fournis par ces nouvelles données topographiques.

Alors que beaucoup d'articles font la part belle aux techniques de traitement informatique des données LiDAR ${ }^{1}$, nous avons voulu insister ici sur les stratégies et méthodes de prospection mises en œuvre sur le terrain, pour localiser et interpréter les indices que livre le laser scanneur aéroporté. Il peut être utile, en particulier pour ceux qui se lancent dans l'archéologie forestière, d'exposer de façon très pratique la chaine opératoire de ces approches en surface et d'insister sur l'importance des vérifications au sol pour interpréter les images numériques, en mesurer aussi les limites. Or la forêt de Tronçais a bénéficié d'assez nombreuses approches archéologiques, antérieurement à l'acquisition LiDAR de 2016. Il est donc possible de comparer la carte archéologique avant et après le passage au laser, de faire la part des avancées, voire des révélations dues à cette technique, mais aussi de ce qui nous échappe encore.

${ }^{1}$ Voir notamment dans ce numéro spécial les développements sur le traitement semi-automatique des données dans l'article de R. de Matos Machado et al. Voir aussi par exemple Fernandez-Diaz et al. 2014 ou Trier, Holger Pilø 2015. 


\section{Cadre géographique}

La forêt domaniale de Tronçais se trouve au centre de la France, dans le nord-ouest du département de l'Allier, en limite septentrionale de la région Auvergne-Rhône-Alpes. Environné par le bocage bourbonnais, le massif forestier couvre une surface de 10600 hectares, répartis sur le territoire de dix communes (fig. 1).

Géologiquement, cette zone se trouve sur les premiers contreforts du Massif central, non loin de terrains marno-calcaires jurassiques. En majorité issus des formations du Trias surmontées par des dépôts de l'Éocène, les substrats se composent de grès, d'argile et de sables mais aussi, au sud-est, de roches métamorphiques comme le granite ou le micaschiste (Lulin 1979). À une altitude comprise entre 200 et $350 \mathrm{~m}$, ce terrain présente un faible pendage général vers le nord-ouest, entaillé par deux ruisseaux principaux, affluents du Cher, la Marmande et la Sologne. Les sols y sont majoritairement bruns acides ou lessivés, souvent pauvres en réserves minérales, assortis de sols d'érosion sur les pentes et rebords de plateaux, de sols colluviaux sableux ou caillouteux au creux des vallons (Bonfils 1970, Bourdet 2000).

Labélisé «forêt d'exception » par l'Office national des forêts (ONF) en 2018, le massif de Tronçais est considéré comme l'une des plus belles chênaies d'Europe. Si les chênes représentent $81 \%$ de son peuplement, on y trouve aussi des hêtres $(10 \%)$, des pins sylvestres $(7 \%)$ et d'autres essences $(2 \%)^{2}$. C'est une forêt ancienne ${ }^{3}$, dont les contours actuels n'ont guère changé depuis la réformation orchestrée par Colbert au XVII ${ }^{\mathrm{e}}$ siècle, comme le montre la plus ancienne carte du secteur (Fleury 1665).

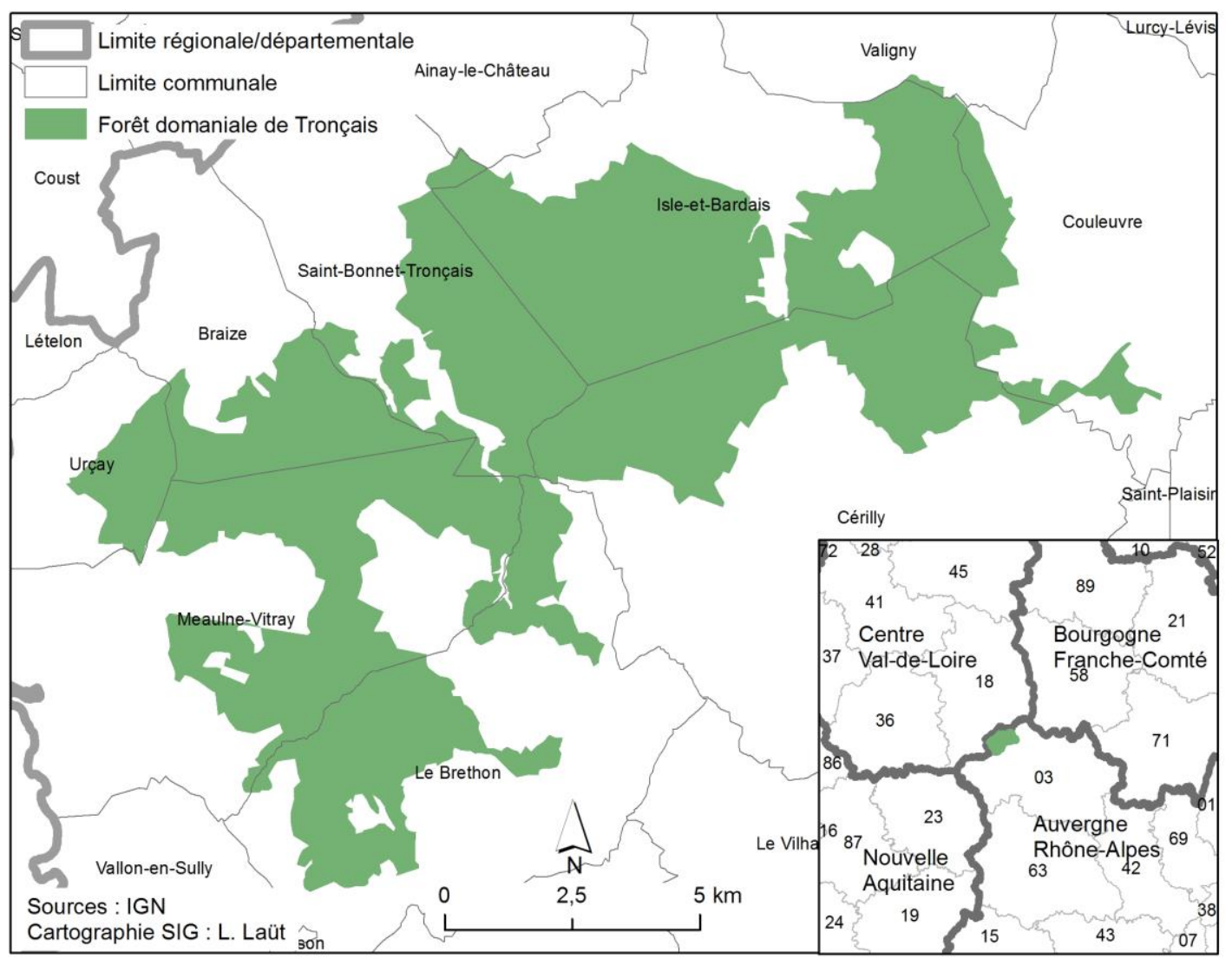

Figure 1. Carte de localisation de la forêt domaniale de Tronçais (L. Laüt).

\footnotetext{
${ }^{2}$ Pour plus de détails sur cette forêt domaniale, voir en particulier le portail qui lui est consacré sur le site de l'ONF (http://www1.onf.fr/enforet/troncais), et le site de la Société des Amis de la forêt de Tronçais (http://www.amis-troncais.org/).

3 Une forêt est dite "ancienne» quand elle est établie sur un sol dont la continuité de l'occupation forestière est attestée depuis plusieurs siècles sans interruption (Bergès, Dupouey 2017). Voir aussi le site web du programme GIP-Ecofor «Cartofora » (http://www.gip-ecofor.org/cartofora/).
} 


\section{Historique des approches archéologiques jusqu'en 2015}

\subsection{Prospections pédestres et géophysiques}

Depuis les années 1960, le massif de Tronçais a fait l'objet de prospections archéologiques menées par des érudits locaux, notamment Élie Bertrand. Ce dernier a ainsi repéré une centaine de sites galloromains, mais aussi des parcours de voies et chemins présumés antiques, des carrières et autres anciens étangs, présentés dans plusieurs publications (dont Bertrand 1980, 1983, 2000, Piboule et Bertrand 1995). En 2000 et 2001, dans le cadre du «PCR-Berry», de nouvelles prospections ont permis de vérifier ses observations, de caractériser plus précisément les vestiges et de compléter un peu la carte archéologique (Laüt 2004, 2007, Gandini, Dumasy et Laüt 2013, Laüt et al. 2014).

Signalons aussi qu'une prospection géophysique a été menée en 2008 par Cécilia Bobée et Gabriel Caraire sur le site des Petits Jardins à Isle-et-Bardais, alors en cours de fouille. Sur une surface de $8100 \mathrm{~m}^{2}$, l'intervention fut difficile en raison du couvert forestier, mais elle permit de révéler l'emplacement de structures enfouies, en complément de celles qui avaient déjà été mises au jour (Caraire 2008, Laüt 2012 : 189-191).

\subsection{Sondages et fouilles}

Des sondages ont été menés en 2001 sur trois sites gallo-romains de la forêt de Tronçais : un atelier de tuilier à Isle-et-Bardais, la mare d'un habitat au Brethon et un bâtiment rural à Saint-BonnetTronçais (Laüt 2004 : 62-72, Laüt et al. 2014 : 216-218). Ces investigations ont permis de constater l'état de conservation relativement bon des vestiges antiques dans ce secteur du territoire de Bituriges Cubes, protégé depuis de nombreux siècles par la forêt.

Ensuite, une fouille programmée a été lancée en 2002 sur le site des Petits Jardins à Isle-et-Bardais (Laüt 2011, Laüt 2012, Laüt 2013a, b, Laüt et al. 2014, Laüt 2015). Un lieu de culte antique y fut aménagé au $\mathrm{I}^{\text {er }}$ siècle apr. J.-C., en même temps que fleurissaient sur la zone des habitats ruraux modestes, fermes ou petites villae. Dans un second temps, bien après l'abandon du sanctuaire intervenu sans doute au III $^{\mathrm{e}}$ siècle, un atelier de tuilier fut aménagé sur le site au haut Moyen Âge, avec ses deux fours installés dans les ruines d'un des temples.

\subsection{Analyses paléoenvironnementales et environnementales}

Entre 2001 et 2005, des analyses pédologiques, anthracologiques et floristiques ont aussi été menées par des chercheurs de l'INRA, sur une sélection de sites gallo-romains, afin de mesurer l'impact de ces occupations anciennes sur les sols et la biodiversité actuels. Ces travaux ont permis de montrer que dans un rayon de 100 à $200 \mathrm{~m}$ autour des habitats, la composition chimique et la texture des sols étaient modifiées, mais aussi que la densité et la diversité de la végétation augmentaient (Bourdet 2000, Humbert 2002, Laüt 2007, Dambrine et al. 2007, Diedhiou et al. 2009, 2010, Laüt et al. 2014).

Des analyses polliniques ont aussi été pratiquées par Hervé Richard sur quelques prélèvements, à l'occasion des sondages et fouilles, suggérant l'existence de productions céréalières aux abords de certains habitats ruraux, dans une ambiance forestière déjà assez marquée (Laüt et al. 2014 : 221 ).

Enfin, en 2011, une étude centrée sur le bassin-versant de Saint-Bonnet-Tronçais a permis de relever d'anciens chemins et des aménagements de berges de ruisseaux, qui ont pu servir à protéger et mettre en valeur les sols (Giosa 2011).

\subsection{Bilan des connaissances avant l'acquisition LiDAR}

À la veille de l'acquisition LiDAR de 2016, 145 sites archéologiques étaient répertoriés dans le périmètre de la forêt domaniale de Tronçais : 2 paléolithiques, 1 néolithique, 113 gallo-romains, 15 médiévaux ou modernes et 14 non datés (fig. 2). Pour l'époque romaine, la mieux représentée ici, nous avons principalement affaire à des vestiges d'habitats ruraux, parfois associés à des traces d'activités 
artisanales (tuilerie, métallurgie). À cela s'ajoutent six parcours de voies anciennes, quelques zones d'extraction, chemins et étangs anciens notés par Élie Bertrand mais non systématiquement vérifiés.

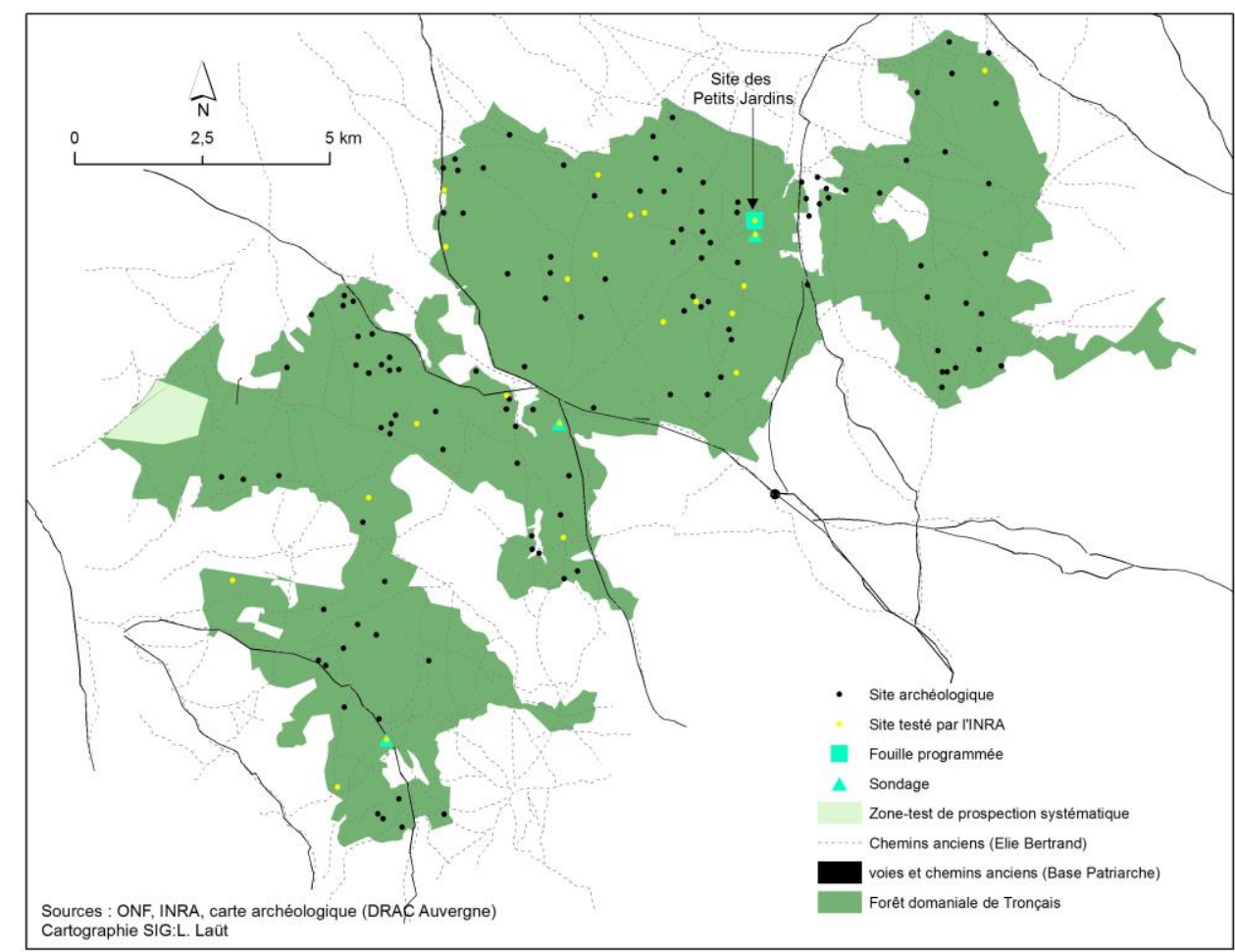

Figure 2. Carte archéologique du secteur, avant l'acquisition LiDAR en 2016 (L. Laüt)

\section{Acquisition et traitement des données LiDAR}

L'acquisition LiDAR a pu être menée grâce à un financement de l'Union européenne, de la du ministère de la Culture (DRAC Auvergne) et de l'ONF. L'opération se fit dans le cadre du programme «LiDARchéo 2016» porté par le Centre régional de l'information géographique (CRAIG), mutualisant 19 projets archéologiques en Auvergne. Sur le secteur de Tronçais, le vol eut lieu le 22 mars 2016, mis en œuvre par la Société Fit-Conseil, prestataire pour l'ensemble du programme auvergnat (fig. 3).
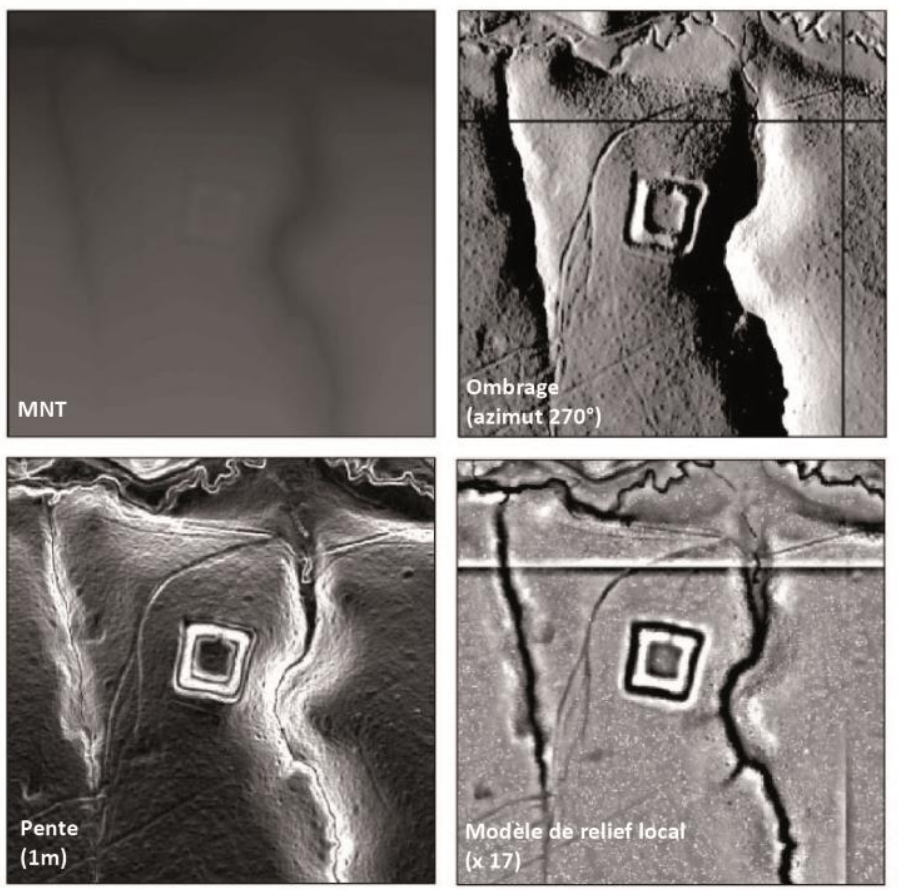

Figure 3. L'emprise de l'acquisition LiDAR sur la forêt domaniale de Tronçais et ses abords (L. Laüt) 
Les données ont ensuite été transmises par le CRAIG à ses différents partenaires, en l'occurrence pour notre secteur la Société des amis de la forêt de Tronçais (SAFT) et l'ONF, en vue de l'exploitation archéologique, mais aussi sylvicole de ces données (Laüt, Guillon 2016, Laüt 2018).

Des vérifications et traitements complémentaires ont ensuite été effectués au sein de l'ONF, notamment par Alain Munoz, Jérôme Mollard et Sophie David. Depuis janvier 2017, nous disposons donc d'un jeu assez complet de données LiDAR (tabl. 1 et fig. 4), couvrant une surface de $197 \mathrm{~km}^{2}$, découpées en 791 dalles de $500 \mathrm{~m}$ de côté, mais aussi assemblées en un seul fichier dans certains cas.

\begin{tabular}{|l|}
\hline \multicolumn{1}{|c|}{ Jeux de données LiDAR sur la forêt de Tronçais et ses abords } \\
\hline Semis de points bruts et classifiés aux formats LAS et ASCII \\
\hline $\begin{array}{l}\text { Modèles numériques de terrain (MNT), d'élévation (MNE), de canopée (MNC) aux formats Arc Grid et } \\
\text { TIN }\end{array}$ \\
\hline MNT avec traitement « Ombrage » : azimuts $45^{\circ}, 90^{\circ}, 135^{\circ}, 180^{\circ}, 225^{\circ}, 270^{\circ}, 315^{\circ}, 360^{\circ}$ \\
\hline MNT avec traitement « Local relief Model » (LRM) : fenêtres $9,9,13,17,21,25$ \\
\hline MNT avec traitement « pente »: facteur Z $1 \mathrm{~m}$ \\
\hline
\end{tabular}

Tableau 1. Jeux de données LiDAR disponibles sur la forêt de Tronçais et ses abords (CRAIG-Fit Conseil 2016, ONF 2017).

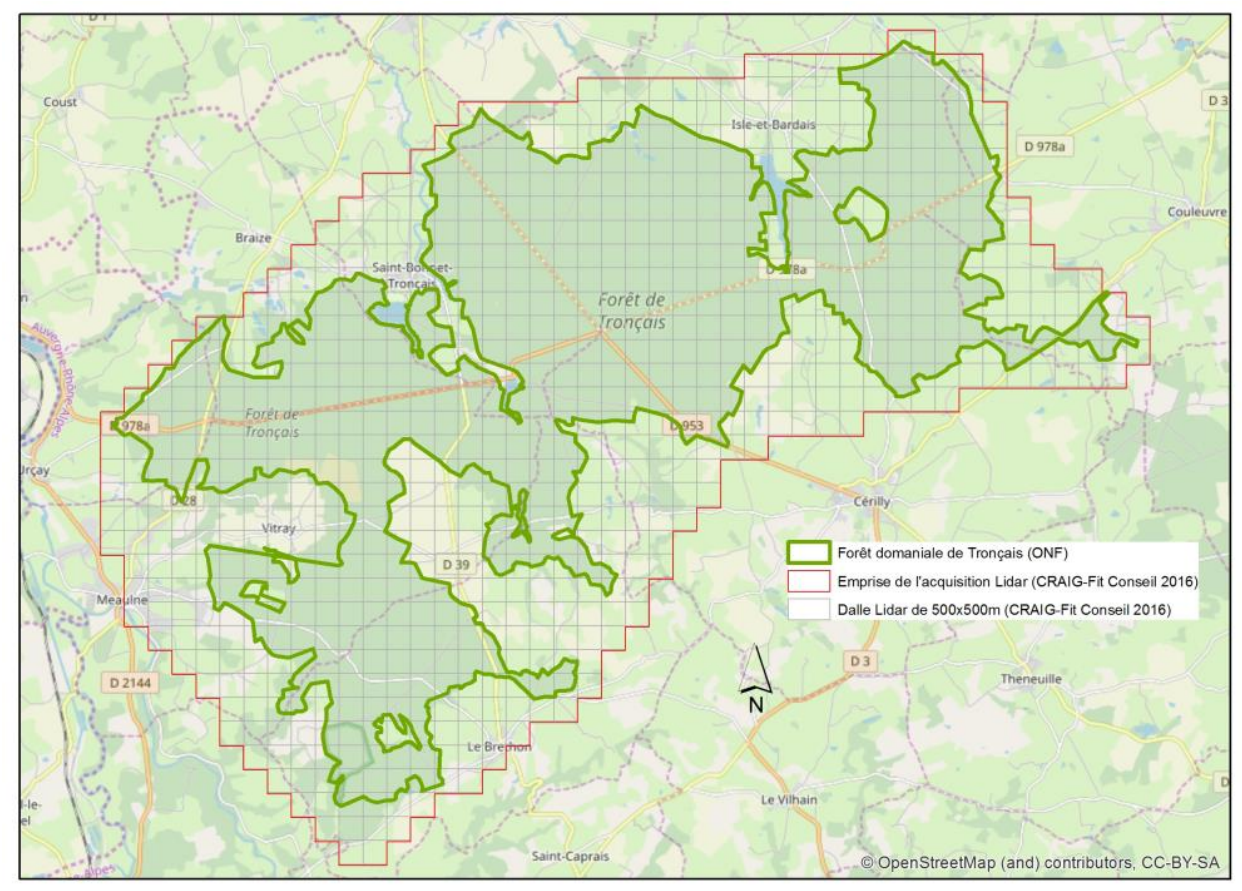

Figure 4 . Quelques-uns des types de traitements des données LiDAR disponibles, où l'on voit une petite fortification quadrangulaire en forêt : le Thureau de Châtelus à Meaulne-Vitray (L. Laüt, données LiDAR : CRAIG-FIT Conseil 2016)

Un premier bilan sur l'intérêt du LiDAR dans la gestion sylvicole du massif a été publié, exploitant principalement les données des différents étages de végétation jusqu'à la canopée (Mollard 2017). Pour l'enquête archéologique, ce sont bien sûr les données concernant le sol qu'il faut considérer. Sur la zone de Tronçais, le lever LiDAR a fourni 20 points $/ \mathrm{m}^{2}$ en moyenne, permettant une approche assez précise des traces d'occupations anciennes. 
Un important travail de vectorisation des anomalies visibles sur les images LiDAR a été mené par Angélique Montès, dans le cadre d'un contrat avec la SAFT, financé par la Drac Auvergne. C'est elle qui a relevé les anomalies polygonales et linéaires, auxquelles nous avons ajouté deux séries d'anomalies ponctuelles. À ce jour, 513 traces surfaciques, 4884 traces linéaires et 3203 traces ponctuelles ont été cartographiées sous SIG (tabl. 2 et fig. 5).

\begin{tabular}{|c|c|c|}
\hline Formes des anomalies sous SIG & Catégorie & Nombre d'entités \\
\hline \multirow[t]{2}{*}{ Polygones } & Zones d'extraction & 283 \\
\hline & Anciennes constructions & 230 \\
\hline \multirow{2}{*}{ Lignes } & Voies et chemins anciens & 200 (70 km linéaires) \\
\hline & Parcellaires anciens & 3810 (490 km linéaires) \\
\hline & Mares et autres dépressions ponctuelles & 508 \\
\hline & Anciennes plateformes de charbonnage & 2695 \\
\hline
\end{tabular}

Tableau 2. Les différentes catégories d'indices vectorisés sous SIG à partir des images LiDAR (A. Montès, L. Laüt)
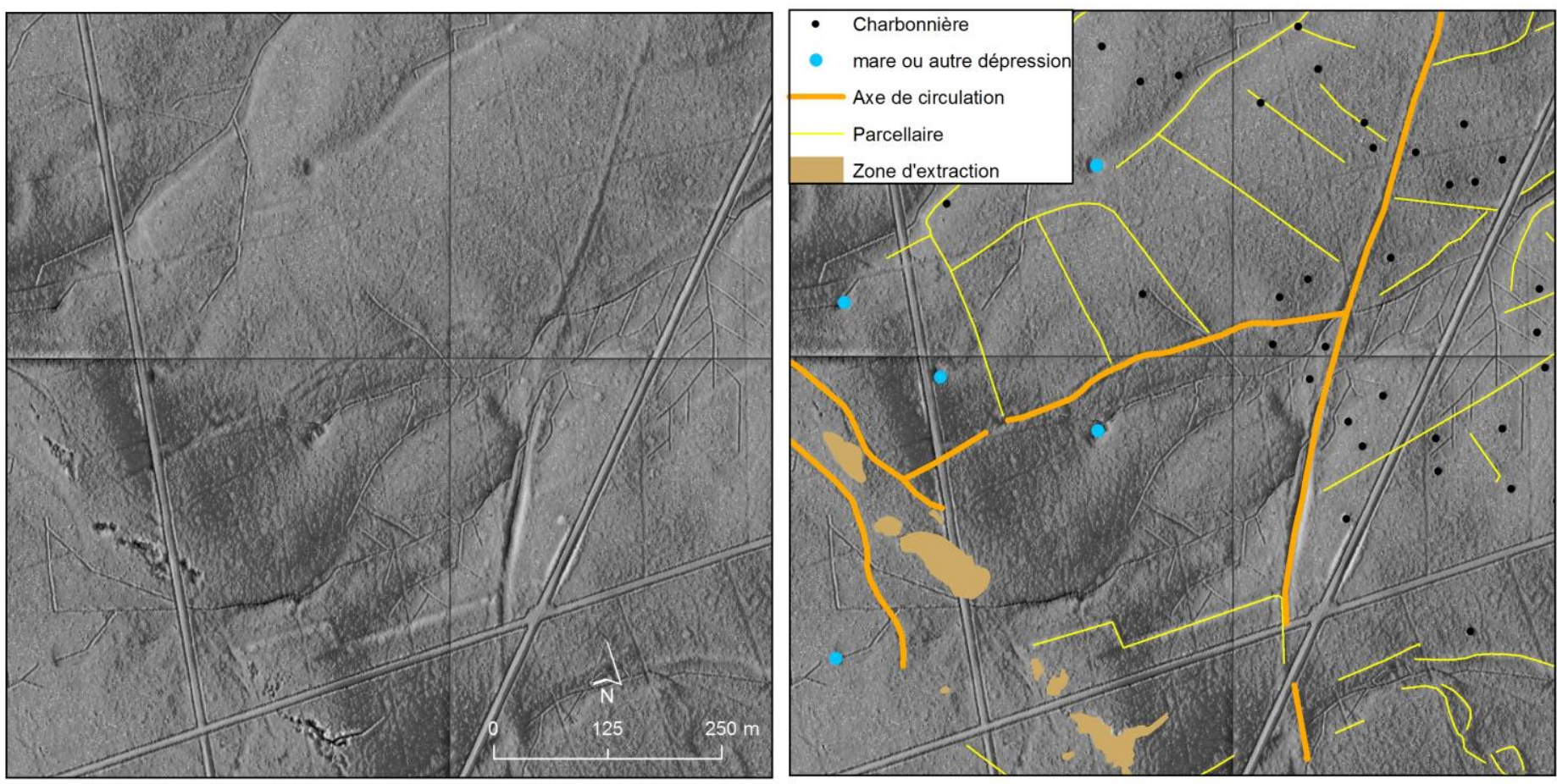

Figure 5. Exemple de relevé des anomalies visibles sur les images LiDAR (LiDAR : CRAIG Fit-Conseil 2016, relevé : A. Montès, L. Laüt) 


\section{Les prospections de 2017-2019}

\subsection{Travaux préliminaires}

La première étape du travail consiste à rassembler la documentation sur la zone étudiée. Pour caractériser le cadre naturel, nous disposons notamment de la carte géologique au 1/50 000 du BRGM $\left(\mathrm{n}^{\circ} 572\right.$ et 573$)$, d'une carte pédologique (L. Humbert [INRA], d'après Bonfils 1970) et de la carte des stations forestières et peuplements de l'ONF (mise à jour d'après Delaunay, Nebout et Beaufils 1991). Concernant les données historiques, si les documents d'archives sont hélas peu nombreux, une série d'articles a été publiée dans les revues locales, en particulier le bulletin de la Société des amis de la forêt de Tronçais ainsi que des études monographiques (notamment Raffignon et Chevalier 1913, Chevalier 1940, Piboule et Bertrand 1995, Romane 2001, Auclair, Woronoff 2005, ou encore Bessard 2018). En outre, différentes cartes anciennes sont riches d'informations, pour les XVII ${ }^{\mathrm{e}}$ (Fleury 1665), XVIII $^{\mathrm{e}}$ (Cassini, feuilles 11 et 50) et $\mathrm{XIX}^{\mathrm{e}}$ siècles (État-Major, 134, 135, 145). Quant aux données archéologiques, elles proviennent de la base Patriarche du ministère de la Culture et de notre base de données compilant les informations fournies par les érudits locaux et les résultats de nos précédentes prospections.

En parallèle, ont été demandées et obtenues des autorisations de la DRAC (prospections pédestres, utilisation d'un détecteur de métaux) et de l'ONF (circulation sur les chemins forestiers habituellement interdits au public), mais aussi des subventions de la DRAC et du Conseil départemental, pour mener à bien ce programme de recherches.

\subsection{Stratégie et procédures de terrain}

Le choix a été fait d'intervenir en priorité sur les sites inédits, en répartissant les interventions sur l'ensemble du massif forestier, dès la première campagne de prospection. Nous avons ainsi pu cerner assez rapidement le potentiel de découvertes que nous offraient les images LiDAR et tester les différents secteurs d'étude.

Une petite équipe de deux à quatre personnes s'avère largement suffisante pour de telles vérifications ponctuelles ${ }^{4}$. Dans une forêt de feuillus, les opérations doivent se faire en période de repos végétatif, afin de bénéficier des meilleures conditions de visibilité, lorsque les arbres ont perdu leurs feuilles et que le sol n'est pas trop encombré de végétation. Quant à l'équipement emporté sur le terrain, il concerne l'enregistrement des données (fiches, tablette et batterie externe de secours), la collecte d'artefacts (sacs, détecteur de métaux, binette) et enfin la prise de clichés (appareil photo de la tablette, mire, boussole et flèche Nord).

Pour le repérage des anomalies sur le terrain, nous avons utilisé l'application Avenza Maps ${ }^{\circledR}$ (anciennement pdf Maps $^{\circledR}$ ), qui permet d'importer sur une tablette des fichiers géoréférencés, sur lesquels on peut voir directement sa position (Fig. 6). Nous avons donc travaillé avec une série de cartes élaborées sous SIG pour visualiser, au fil de notre progression et selon les besoins, différents types d'images LiDAR et autres fonds de cartes (géologie, pédologie, cartes anciennes, etc.). Cela s'avère très utile pour aller d'un indice à l'autre dans de très grandes parcelles forestières où il est difficile de se repérer. En outre, cette application fonctionnant hors connexion à Internet, son utilisation n’est pas tributaire des difficultés d'accès aux réseaux, fréquentes en sous-bois.

\footnotetext{
${ }^{4}$ Que soient ici remerciés les prospecteurs bénévoles qui sont intervenus sur le terrain à nos côtés : Jean Archambault, Patrick Defaix, Claude Jimenez, Corinne Opresco, Agathe Riou, Florie Salvaya, Alain, Olivia et Raphaël Taccoen, Claude Terrade et JeanGuy Voisine.
} 


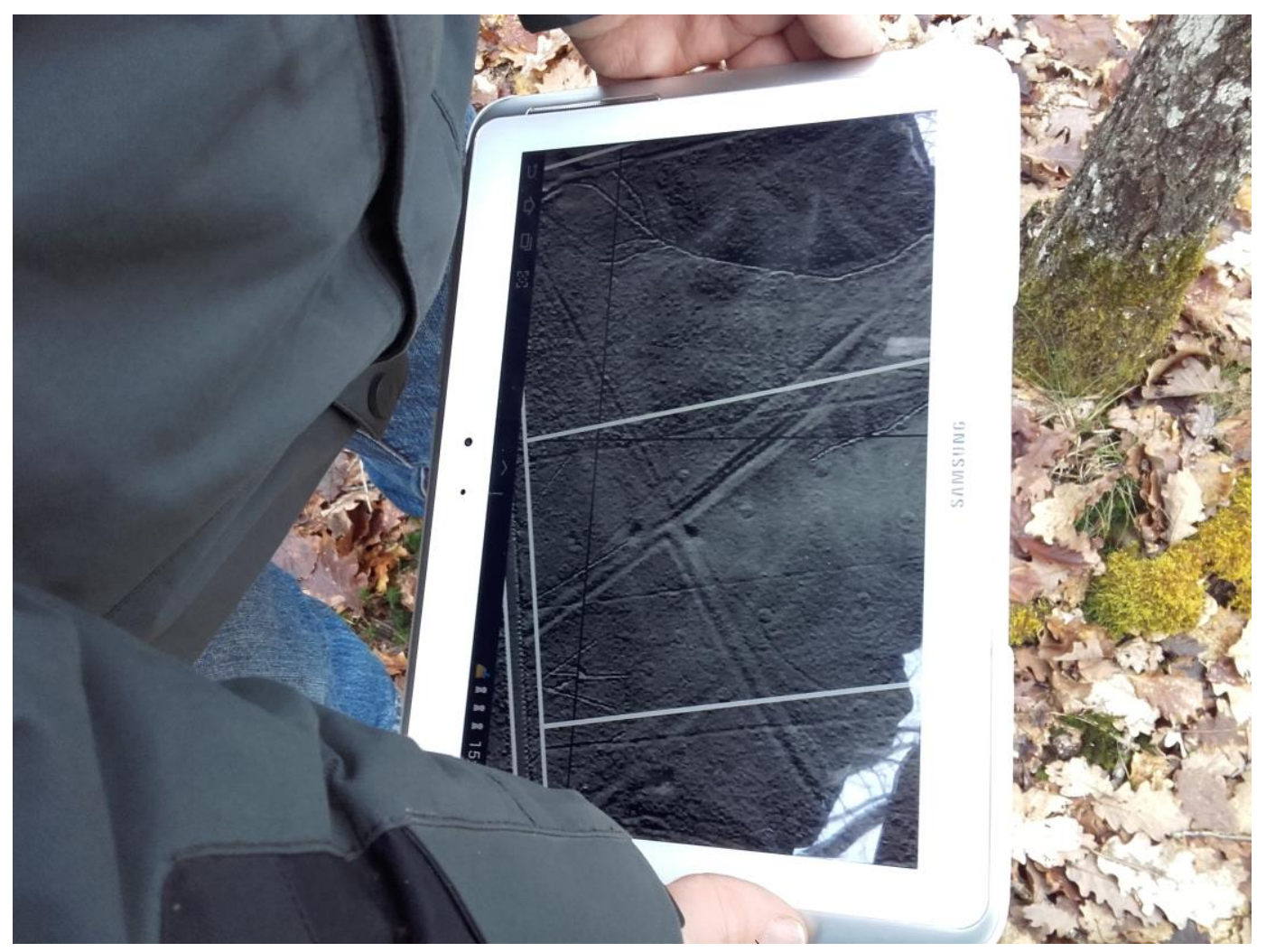

Figure 6. Utilisation de la tablette sur le terrain, pour visualiser l'image LiDAR et être géolocalisé en permanence sur cette image (L. Laüt).

Une fois l'anomalie LiDAR atteinte, une observation à vue est menée, pour apprécier la nature des reliefs et rechercher des artefacts. Une fiche «parcelle» est remplie sur un support papier, qui renseigne sur l'état de la parcelle, les conditions de prospection, l'anomalie LiDAR et ce que l'on peut en voir sur le terrain, ou encore le mobilier récolté (fig. 7). Au verso est toujours réalisé un croquis sommaire de la parcelle et de la position des différentes anomalies observées. En revanche, nul besoin de faire des relevés précis sur le terrain, puisque la topographie des reliefs est déjà révélée par les données LiDAR. Il est cependant important de noter tout ce qui peut être observé indépendamment des reliefs visibles (zones de concentration de mobilier, coloration de la terre, objets isolés, etc.).

Il est également possible, via cette application, de marquer des points avec des commentaires ou de faire des clichés géoréférencés. Les données enregistrées peuvent ensuite être exportées, pour être directement visualisées sur Google Earth ou retravaillées sous SIG, après conversion des fichiers .kml en .shp. Mais il est important de conserver l'enregistrement manuel sur fiche papier par sécurité, afin d'éviter la perte définitive de certaines informations en cas de problème informatique avec la tablette...

Concernant les artefacts observés sur chaque anomalie, des choix ont été faits en fonction de critères pratiques (poids, encombrement) et scientifiques (objets caractérisables, datables, analysables, etc.). Certains sont donc laissés sur place après avoir été signalés (moellons et autres blocs taillés, terres cuites architecturales [TCA] indéterminées), d'autres sont recueillis pour étude (fragments de TCA caractérisables, de céramique, de verre, de métal, de meules, scories, culots de forge...).

Rappelons que dans le cadre de ce programme de prospection, une autorisation spéciale nous a été accordée par la DRAC Auvergne-Rhône-Alpes, pour utiliser un détecteur de métaux. Sachant que tous les sites archéologiques déjà publiés dans ce secteur forestier ont fait l'objet de pillage par des détectoristes clandestins, nous privant de précieuses informations sur leur chronologie et leur nature, nous avons préféré intervenir avant eux sur les sites inédits révélés par les images LiDAR. Il est dommage d'en arriver là car ce faisant, nous perturbons nous aussi quelque peu la stratigraphie des sites. Mais cette politique du moindre mal permet de sauver des données archéologiques qui auraient de toute façon disparu après la publication des découvertes. C'est d'ailleurs par souci de préserver 
encore un peu ce patrimoine archéologique que cet article ne présente ni carte des nouveaux sites, ni indication géographique pour les exemples figurés...

\begin{tabular}{|c|c|}
\hline \multicolumn{2}{|r|}{ Informations manuscrites sur fiche } \\
\hline \multirow{5}{*}{ 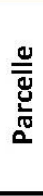 } & Référence ONF \\
\hline & Référence cadastrale \\
\hline & Commune \\
\hline & Lieu-dit \\
\hline & Etat de la parcelle \\
\hline \multirow{7}{*}{ 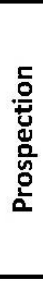 } & Session \\
\hline & Date \\
\hline & Heure \\
\hline & Prospecteurs \\
\hline & Présence de chablis (case à cocher) \\
\hline & Ensoleillé / couvert (cases à cocher) \\
\hline & Sol sec / sol humide (cases à cocher) \\
\hline \multirow{10}{*}{ 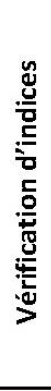 } & $\mathrm{N}^{\circ} \mathrm{d}^{\prime}$ indice \\
\hline & $\mathrm{N}^{\circ}$ de site (si déjà connu) \\
\hline & Description de l'anomalie Lidar \\
\hline & Observations sur le terrain \\
\hline & Utilisation du détecteur de métaux (case à cocher) \\
\hline & Prise de clichés (case à cocher) \\
\hline & Interprétation \\
\hline & Datation \\
\hline & Remarques \\
\hline & Croquis de localisation des indices dans la parcelle \\
\hline
\end{tabular}

\begin{tabular}{|c|}
\hline \multicolumn{1}{|c|}{ Informations saisies sur tablette } \\
\hline Points repères (fichiers .KML) \\
\hline Informations attachées à chaque point : \\
- $\quad N^{\circ} n^{\prime}$ indice \\
- Descriptif \\
- Clichés (géo-référencement automatique) \\
- Coordonnées $\mathrm{X}, \mathrm{Y}$ (automatique) \\
- $\quad$ Date, heure (automatique) \\
\hline
\end{tabular}

\begin{tabular}{c} 
Collecte d'artefacts \\
(TCA, céramique, verre, scories, éléments \\
métalliques, etc.) \\
Prélèvements \\
(sédiment, pierre, charbon, etc.) \\
\hline Sacs portant le $\mathrm{n}^{\circ}$ de l'indice
\end{tabular}

Figure 7. Tableaux récapitulatifs des données récoltées sur le terrain.

\subsection{Traitement des données}

L'ensemble des informations recueillies sur le terrain est ensuite enregistré dans une base de données qui comporte deux tables distinctes, consacrées respectivement à la parcelle prospectée et à l'indice LiDAR vérifié. Cet enregistrement s'accompagne d'une cartographie sous SIG permettant de visualiser les résultats, mais aussi de procéder à un certain nombre d'analyses spatiales. Quant aux artefacts ou échantillons recueillis sur le terrain, ils sont classés par matériaux, identifiés, comptés et, pour certains, dessinés, photographiés ou radiographiés (fig. 8).

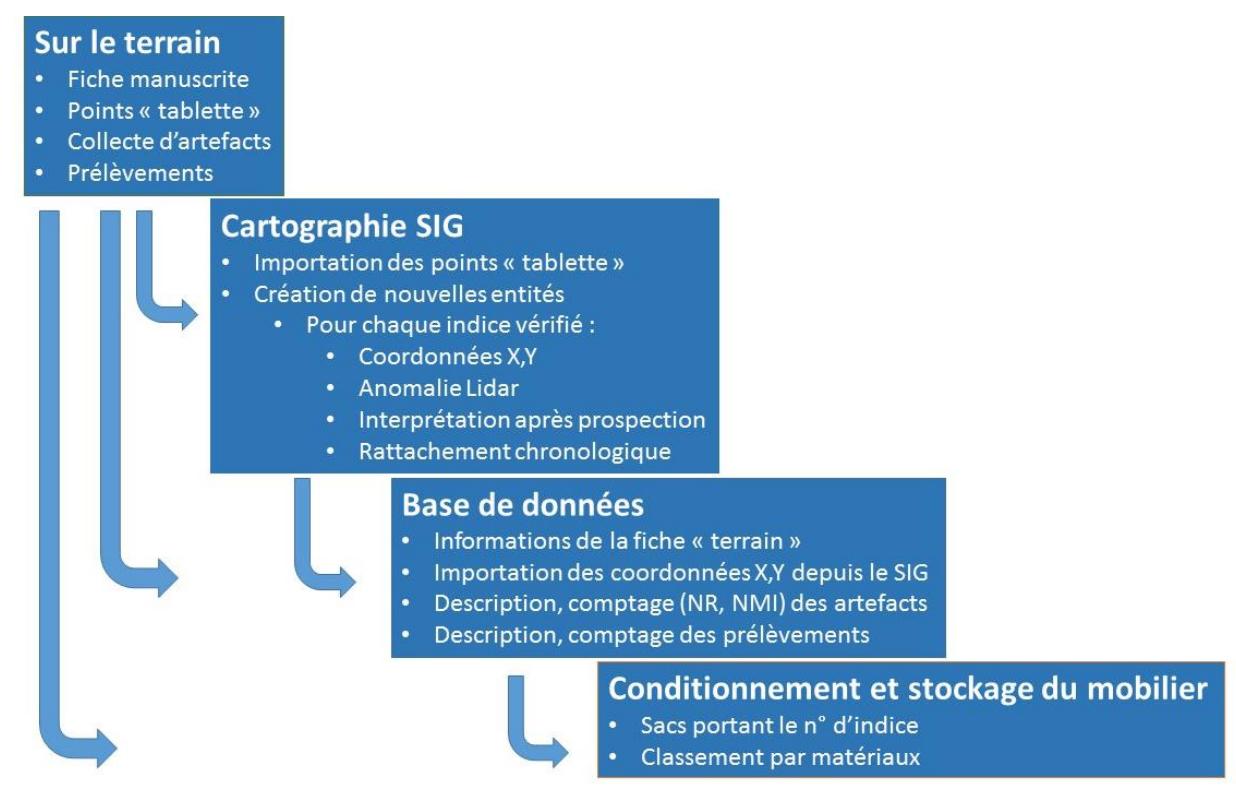

Figure 8. Schéma des différentes étapes du travail, pour l'enregistrement des données archéologiques (L. Laüt) 


\subsection{Bilan des prospections en juin 2019}

Depuis janvier 2017, 288 parcelles forestières sur 442 ont fait l'objet de vérifications sur le terrain, en 30 journées de prospections automnales ou hivernales. Ce sont donc près de 10 parcelles qui ont été vérifiées par jour en moyenne, ce chiffre pouvant varier considérablement d'un jour à l'autre, en fonction du nombre d'indices à contrôler et des découvertes faites sur le terrain. Rappelons qu'il ne s'agit pas d'une prospection systématique, mais d'une vérification, dans chaque parcelle, des principaux indices LiDAR (fig. 9).

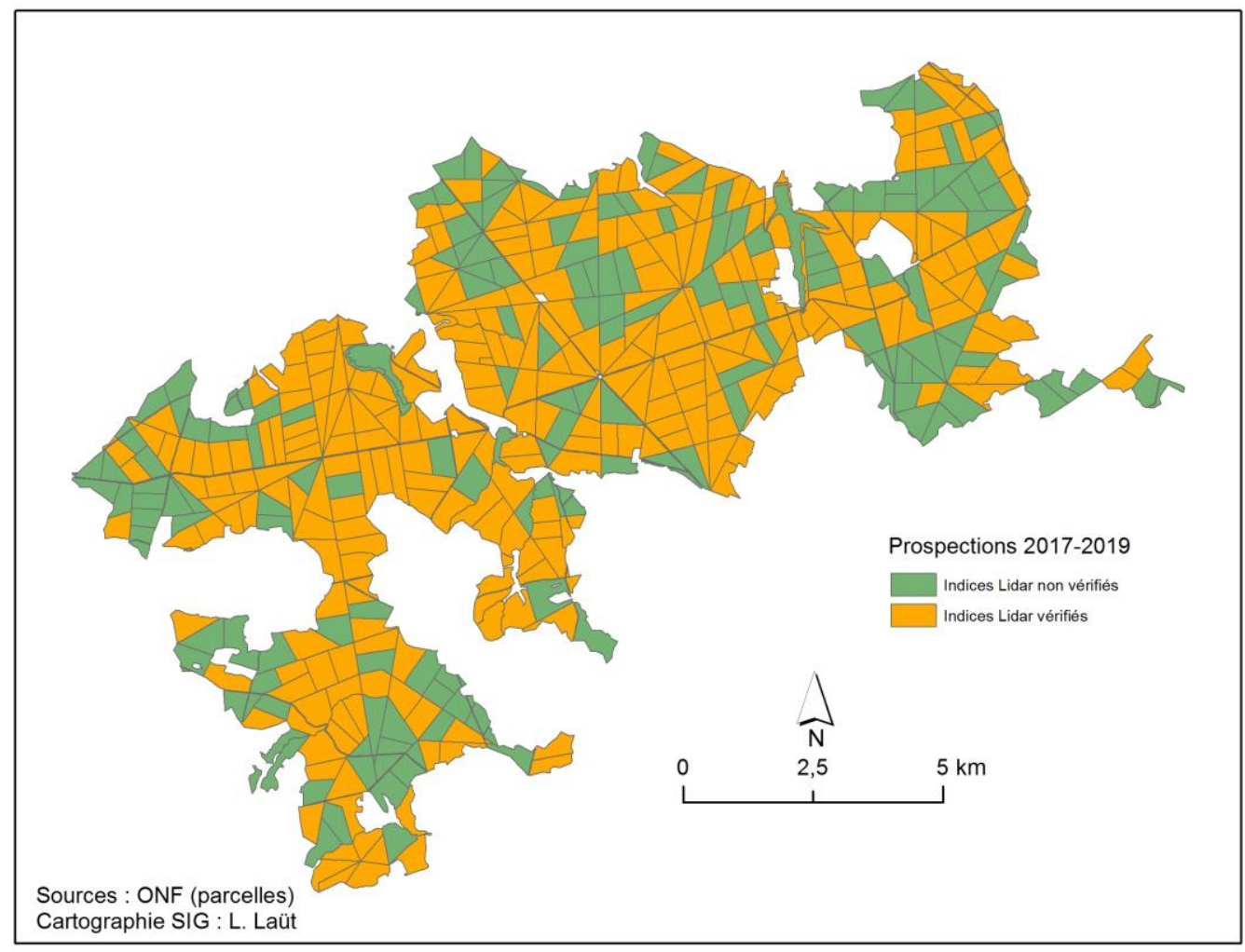

Figure 9. Carte des parcelles prospectées entre janvier 2017 et avril 2019 (L. Laüt)

À ce jour, 868 indices ont fait l'objet d'une vérification. Environ $10 \%$ d'entre eux se sont révélés être des reliefs naturels (affleurements rocheux, glissements de terrain), des éléments végétaux (amas de bois morts, ronciers) ou des traces de travaux forestiers contemporains. Dans $90 \%$ des cas, nous avons donc affaire à des reliefs indiquant différentes formes d'occupations anciennes (fig. 10). Toutefois, sur près de $60 \%$ de ces indices anthropiques, en dehors d'éventuels blocs taillés, aucun artefact n'a été recueilli, ce qui est souvent le cas sur les structures parcellaires, les dépressions ponctuelles (anciennes mares?) ou les amas pierreux (tertres ? cabanes? tas d'épierrement?). Mais sur 322 indices, des éléments de TCA, de céramique, de métal ou des déchets métallurgiques permettent de préciser la fonction et la chronologie des occupations.

À l'heure où cet article est rédigé, le programme de prospection 2017-2019 n'est pas terminé et il nous reste à effectuer la session automnale de la dernière campagne. Ainsi devraient pouvoir être contrôlés tous les indices LiDAR et sites archéologiques de la forêt domaniale. 


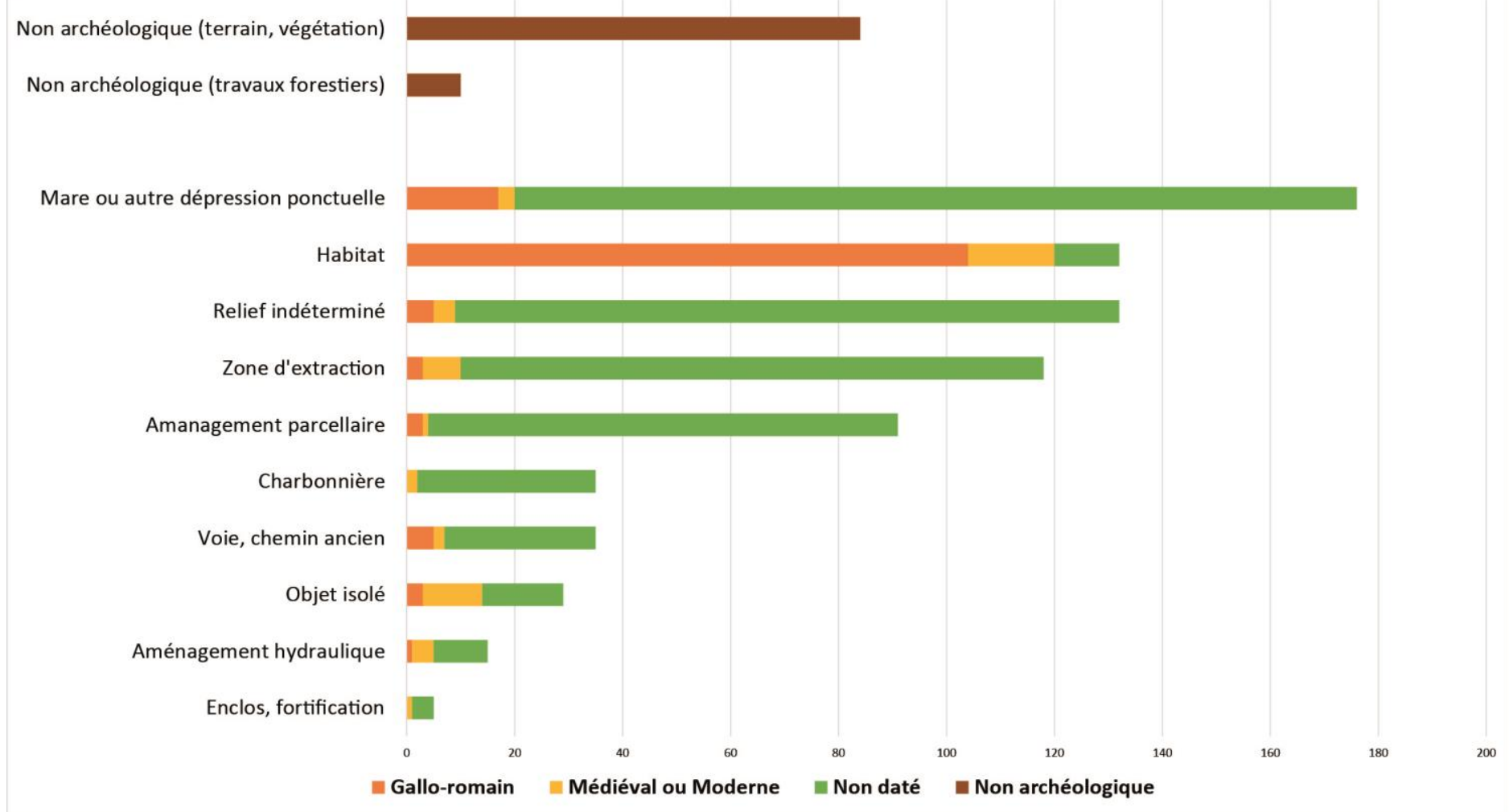

Figure 10. Histogramme des indices vérifiés dans la forêt de Tronçais et ses abords, entre janvier 2017 et avril 2019 (L. Laüt)

\section{Une vision renouvelée des occupations anciennes de la forêt}

\subsection{Ce que l'on voit mieux}

Incontestablement, nous avons une connaissance beaucoup plus précise et complète des reliefs laissés par les anciennes constructions, principalement antiques, dans le massif forestier de Tronçais. Dans leur majorité, ces habitats étaient déjà localisés grâce aux précédentes prospections, mais les images LiDAR ont permis de cerner leur localisation, leur forme et organisation interne de façon beaucoup plus fine. En outre, des constructions encore inconnues ont été révélées, permettant d'augmenter de $35 \%$ le nombre de sites antiques connus à ce jour. Les établissements ruraux de ce secteur se caractérisent par ce qu'Élie Bertrand qualifiait assez justement d' «amas ruiniformes », qui indiquent la présence d'un ou plusieurs bâtiments, mais ne permettent pas d'établir des plans précis (fig. 11). Seulement deux petites fortifications apparaissent sur les images LiDAR, qui étaient déjà connues par ailleurs : l'une, protohistorique, aux abords de la forêt (Motte du Champ de la Tour à Cérilly), l'autre, sans doute médiévale, dans le périmètre de la forêt (Thureau de Châtelus à MeaulneVitray). 

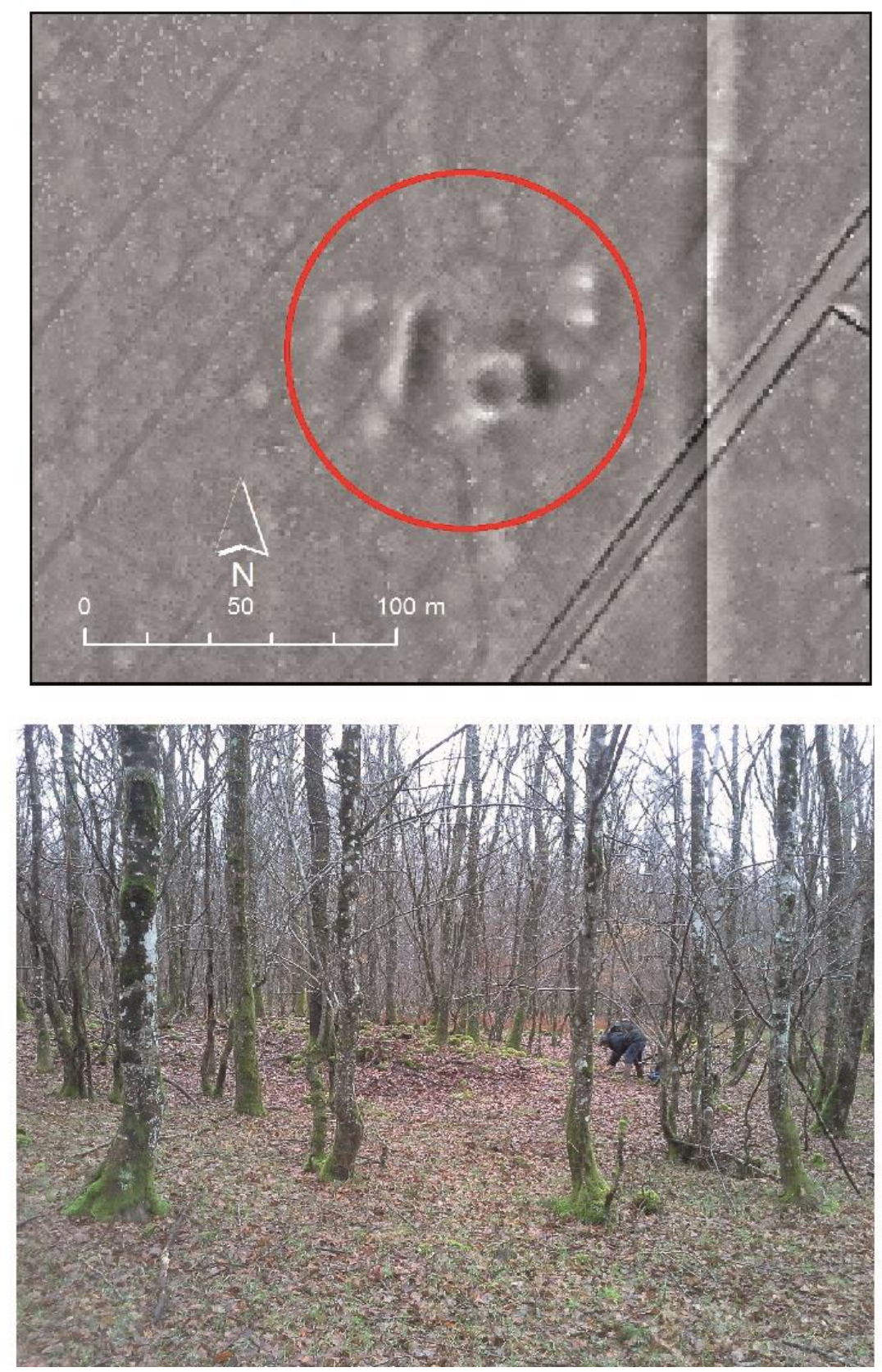

Figure 11. Exemple d'un établissement gallo-romain, tel qu'on le voit sur l'image LiDAR et sur le terrain (LiDAR : CRAIG-Fit Conseil 2016, cliché : L. Laüt).

Les parcours de voies et chemins anciens sont eux aussi bien mieux connus grâce aux images LiDAR. Il ne s'agit pas là de simples précisions sur des parcours déjà connus, mais bien d'une remise en question à peu près complète des tracés préalablement envisagés. Sur les $70 \mathrm{~km}$ linéaires de voies et chemins anciens que montrent les images LiDAR, seuls $16 \mathrm{~km}$ étaient déjà identifiés. En revanche, nombre des hypothèses formulées précédemment, sur la base d'observation de terrain ou de conjectures sur des liaisons entre sites, doivent être aujourd'hui abandonnées. Cela montre qu'il est particulièrement difficile de reconstituer des parcours de voies en sous-bois par de simples repérages visuels, alors que les données microtopographiques du LiDAR offrent une très bonne lisibilité des axes de circulation, dans leur continuité linéaire, sur des centaines de mètres, voire des kilomètres. Sous le massif de Tronçais apparaissent ainsi clairement d'importantes voies avec une chaussée bombée et des fossés latéraux (fig. 12), mais aussi des chemins plus étroits, en relief ou en creux. 

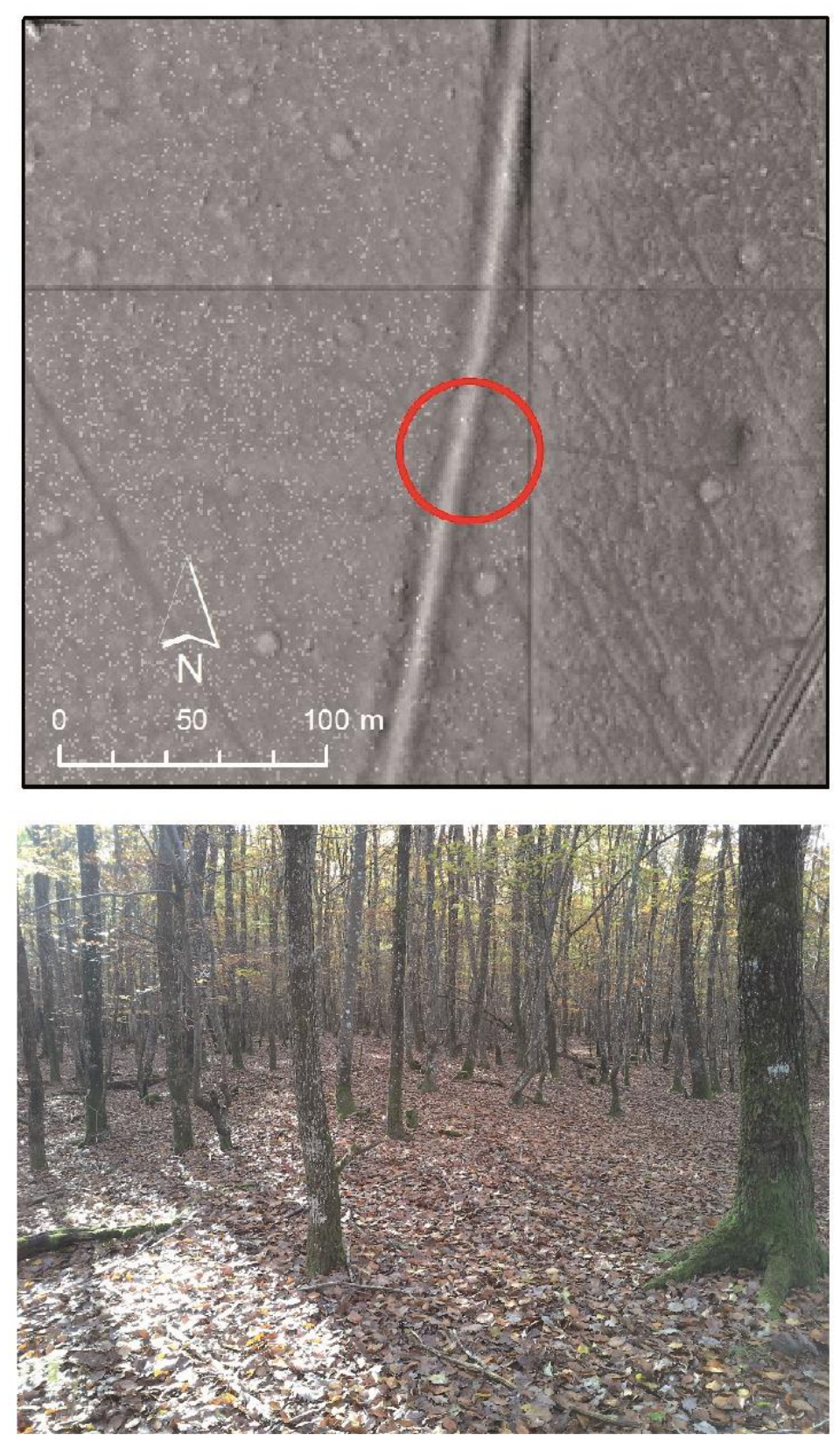

Figure 12. Exemple d'une portion de voie, telle qu'on la voit sur l'image LiDAR et sur le terrain (LiDAR:

CRAIG-Fit Conseil 2016, cliché : L. Laüt)

Une autre catégorie de vestiges est désormais beaucoup mieux cernée, celle des zones d'extraction de matériaux (pierre, sable, argile, minerai de fer). Si certaines carrières avaient déjà été relevées, notamment par Élie Bertrand, aucune enquête archéologique n'avait porté spécifiquement sur ces sites, à une exception près 5 . Or, les zones d'extraction sont particulièrement bien visibles sur les images LiDAR. Elles couvrent une surface totale de 75 hectares sur le secteur étudié et différentes catégories peuvent être distinguées : les carrières en front de taille vertical (souvent récentes), les carrières en tranchées linéaires (fig. 13) et les carrières en cuvettes de surface.

\footnotetext{
${ }^{5}$ Il s'agit d'une carrière d'argile repérée à proximité d'un atelier de tuilier antique, sondé en 2001 et qui avait fait l'objet d'un relevé topographique (Laüt et al. 2014 : 217).
} 

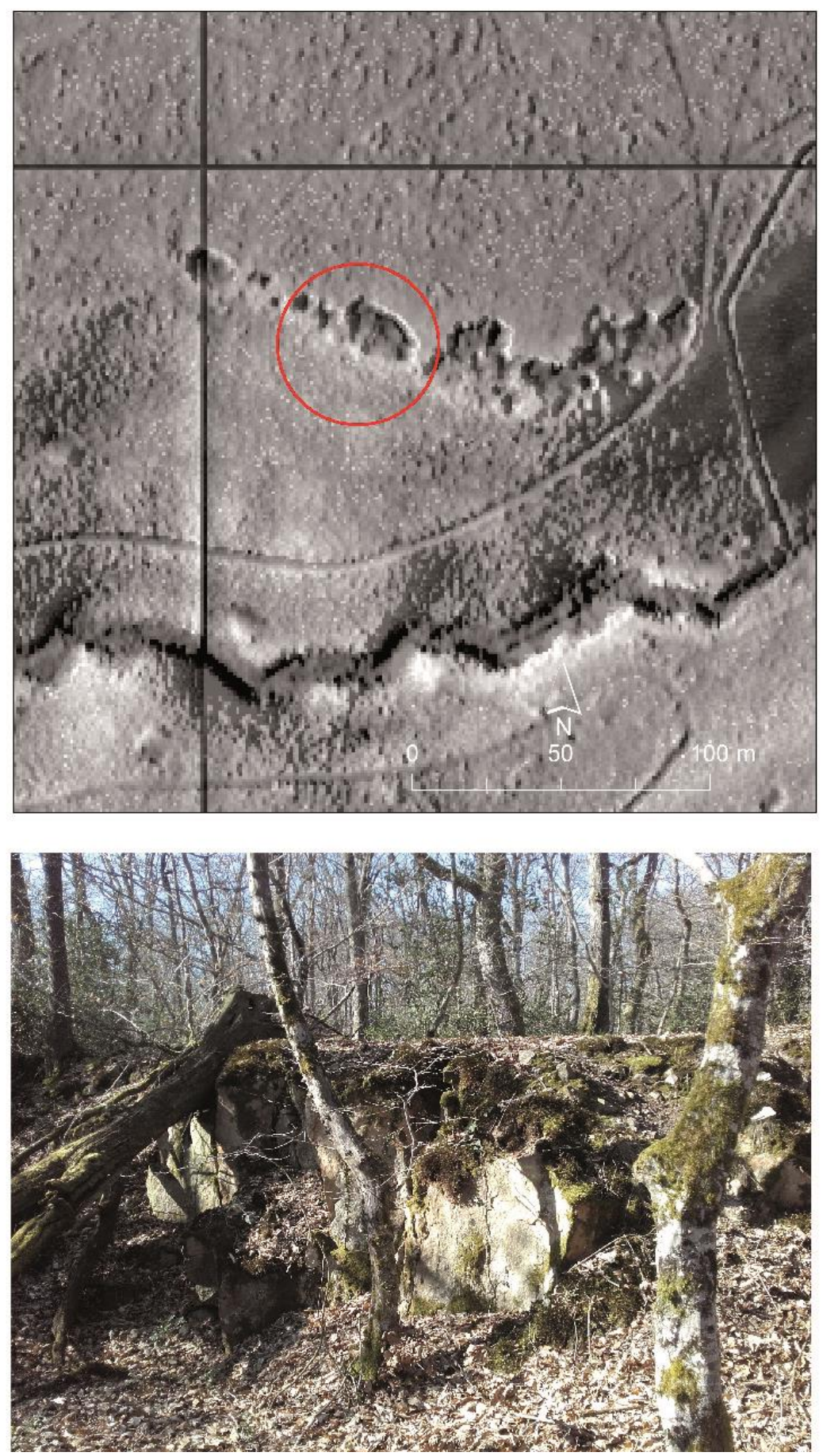

Figure 13. Exemple d'une zone d'extraction, telle qu'on la voit sur l'image LiDAR et sur le terrain (LiDAR : CRAIG-Fit Conseil 2016, cliché : L. Laüt)

Enfin, un élément du paysage ancien est beaucoup mieux cerné, lui aussi : la dépression ponctuelle, souvent interprétée comme une mare. Nous en connaissions déjà une cinquantaine, notamment celles qui jouxtent des constructions antiques, mais aussi quelques-unes plus isolées, parfois associées à du mobilier. Sur les images LiDAR, sont désormais visibles 564 dépressions, qui peuvent correspondre à de petites zones d'extraction, à des mares anciennes ou des collecteurs de fossés de drainage récents, ces différentes fonctions ayant d'ailleurs pu se succéder dans le temps pour un même site (fig. 14). Dans certains cas, les vérifications sur le terrain ont permis de repérer des déchets métallurgiques ou de la céramique, indiquant donc la présence d'habitats ou d'ateliers, sans relief associé à la dépression. 

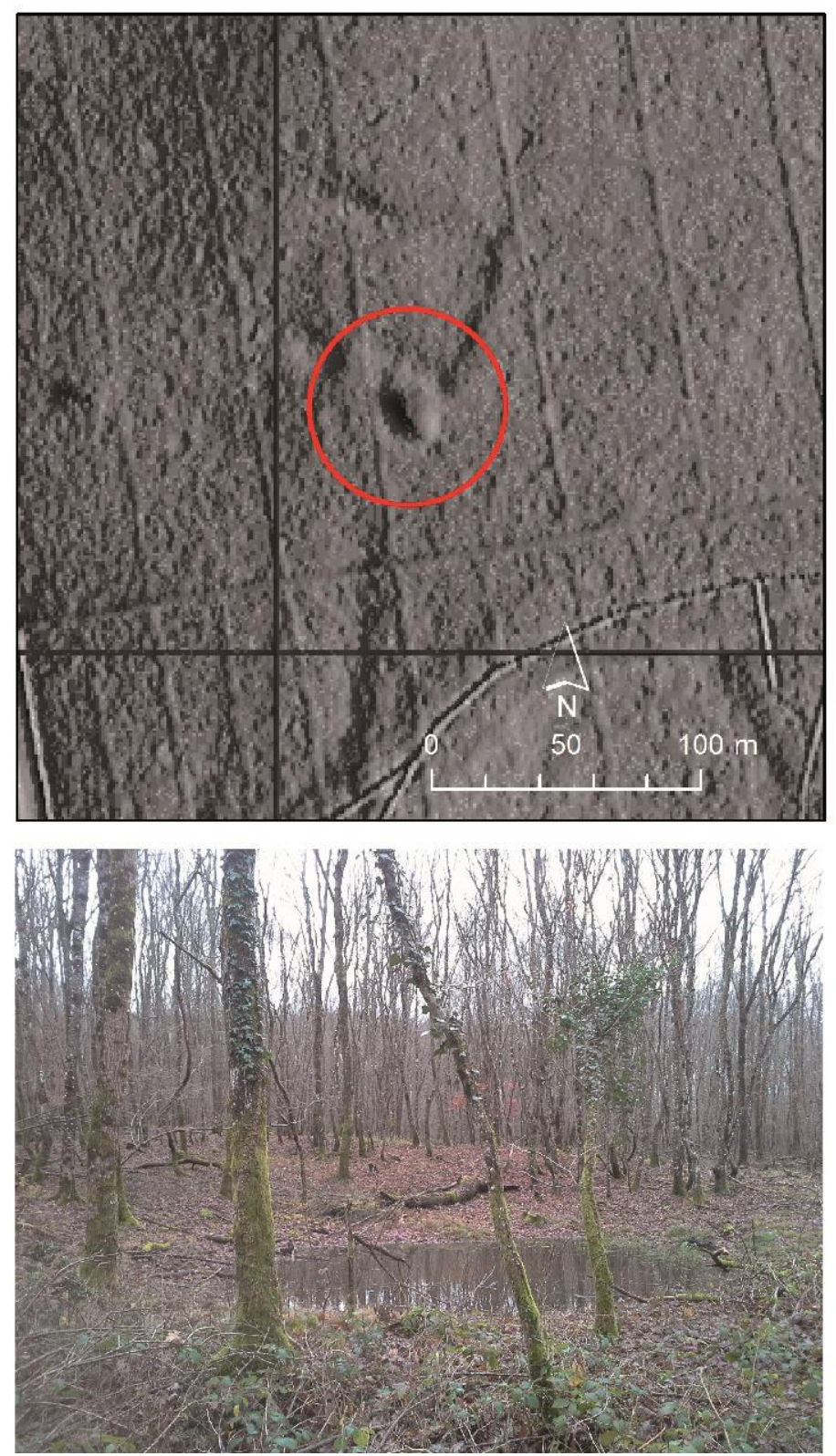

Figure 14. Exemple d'une mare encore en eau associée à un habitat gallo-romain, telle qu'on la voit sur les images LiDAR et sur le terrain (LiDAR : CRAIG-Fit Conseil 2016, cliché : L. Laüt)

\subsection{Ce qui est apparu}

Une composante des paysages anciens nous manquait cruellement avant l'acquisition LiDAR : celle des structures agraires, des limites de champs ou de pâtures, de terrasses, etc. En effet, contrairement à ce que l'on peut observer dans d'autres forêts, où ces délimitations forment des reliefs linéaires bien visibles sur le terrain ${ }^{6}$, de tels reliefs ne sont pas du tout évidents à percevoir en forêt de Tronçais et de fait, n'avaient pas été perçus... L'on en déduisait donc que le contexte dans lequel se sont implantés les habitats antiques devait être largement boisé, avec des cultures uniquement regroupées aux abords immédiats des bâtiments. Cette hypothèse était d'ailleurs corroborée par les résultats des analyses pédologiques et floristiques, qui montraient le rayon limité de l'impact des occupations anciennes sur les sols et la biodiversité actuels. Or, les images LiDAR révèlent pour la première fois une importante quantité de structures agraires ${ }^{7}$ et des contrôles sur le terrain ont permis de confirmer l'existence de ces

\footnotetext{
${ }^{6}$ Par exemple en forêt de Haye en Lorraine (Georges-Leroy 2009, 2011). Voir aussi l'article de M. Georges-Leroy dans ce numéro spécial.

${ }^{7}$ Le même cas de figure s'est présenté par exemple en forêt de Sénart, où le réseau des structures agraires n'est apparu que sur les images Lidar (David 2010, 2011). Voir aussi un diaporama de Sophie David, mis en ligne à la suite de l'Ateliern ${ }^{\circ} 16$ (7 juin 
reliefs souvent discrets mais bien réels. Notre œil commence désormais à mieux les percevoir, cependant seul le modèle numérique de terrain permet d'en saisir la forme géométrique et par là même, le caractère anthropique (fig. 15).

Les réseaux parcellaires identifiés en forêt de Tronçais ne couvrent pas l'ensemble du secteur de façon continue, comme on peut le voir dans d'autres régions ${ }^{8}$. Ici, nous observons une succession de petits ensembles épousant le relief, concentrés dans les zones denses en habitats antiques. Ce dernier constat permet d'ailleurs d'envisager une origine antique pour ces structures agraires. La trame est donc irrégulière et discontinue, adossée par endroits au réseau de circulation et présentant d'assez vastes trous qu'il nous faudra expliquer : correspondent-ils à des zones boisées ? à un effacement des traces parcellaires dû à l'érosion ou au colluvionnement? Quant à l'analyse morphologique de ces réseaux, elle n'en est qu'à ses débuts et nous n'en dirons donc pas plus à ce sujet.
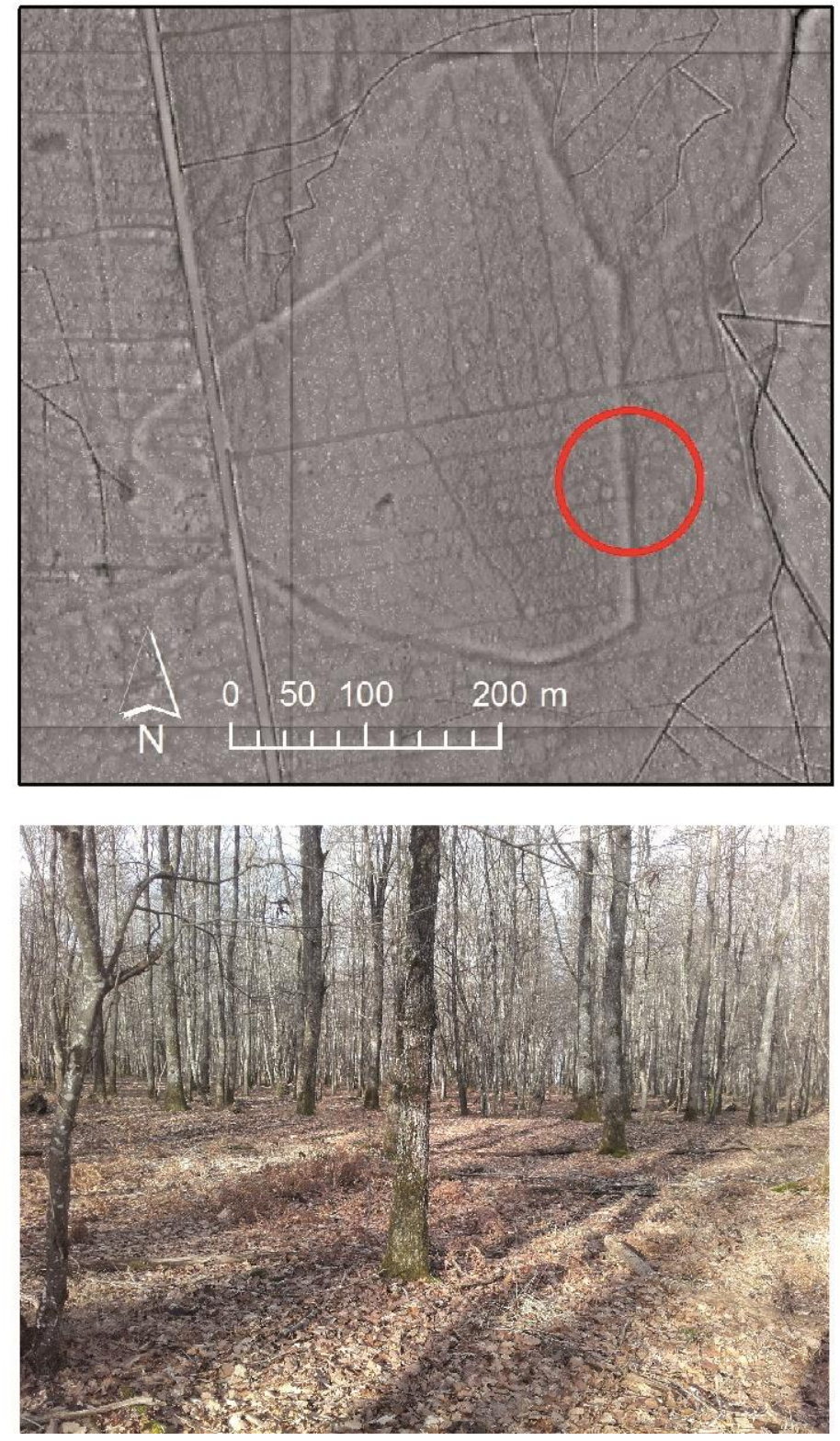

Figure 15. Exemple d'un talus d'enclos parcellaire, tel qu'on le voit sur l'image LiDAR et sur le terrain (LiDAR : CRAIG-Fit Conseil 2016, cliché : L. Laüt)

2016) «Datation des réseaux parcellaires laténiens et gallo-romains», coordonné par François Favory dans le cadre du programme Rurland : https://rurland.hypotheses.org/368.

${ }^{8}$ C'est le cas notamment en forêt de Haye où, avec $647 \mathrm{~km}$ linéaires de structures parcellaires sur $116 \mathrm{~km}^{2}$ de couverture Lidar (Georges-Leroy 2011, tableau 3), la densité de traces est aussi plus forte qu'en forêt de Tronçais (ici, pour rappel, nous avons $490 \mathrm{~km}$ de lignes sur une surface de $197 \mathrm{~km}^{2}$ ). 
D'autres éléments, totalement absents de la carte archéologique, sont apparus sur les images LiDAR : les plateformes de charbonnage ou charbonnières (fig. 16). L'existence de ces structures était bien évidemment déjà connue, car il s'agit d'une activité courante et assez bien documentée, au moins pour les périodes les plus récentes. Mais pour la première fois, les images LiDAR permettent de voir les traces au sol de ces plateformes circulaires d'environ $12 \mathrm{~m}$ de diamètre, pour une hauteur conservée de 20 à $50 \mathrm{~cm}$. Ces plateformes sont particulièrement visibles lorsqu'elles sont aménagées sur des terrains en pente (fig. 16), mais le plus souvent, leurs traces au sol sont très discrètes. Quelque 2700 charbonnières ont ainsi pu être cartographiées à partir des images LiDAR, dont seulement quelquesunes ont fait l'objet de vérifications au sol. Il s'agit là d'un champ de recherche à part entière ${ }^{9}$, mais que nous n'avons que peu exploré pour le moment.
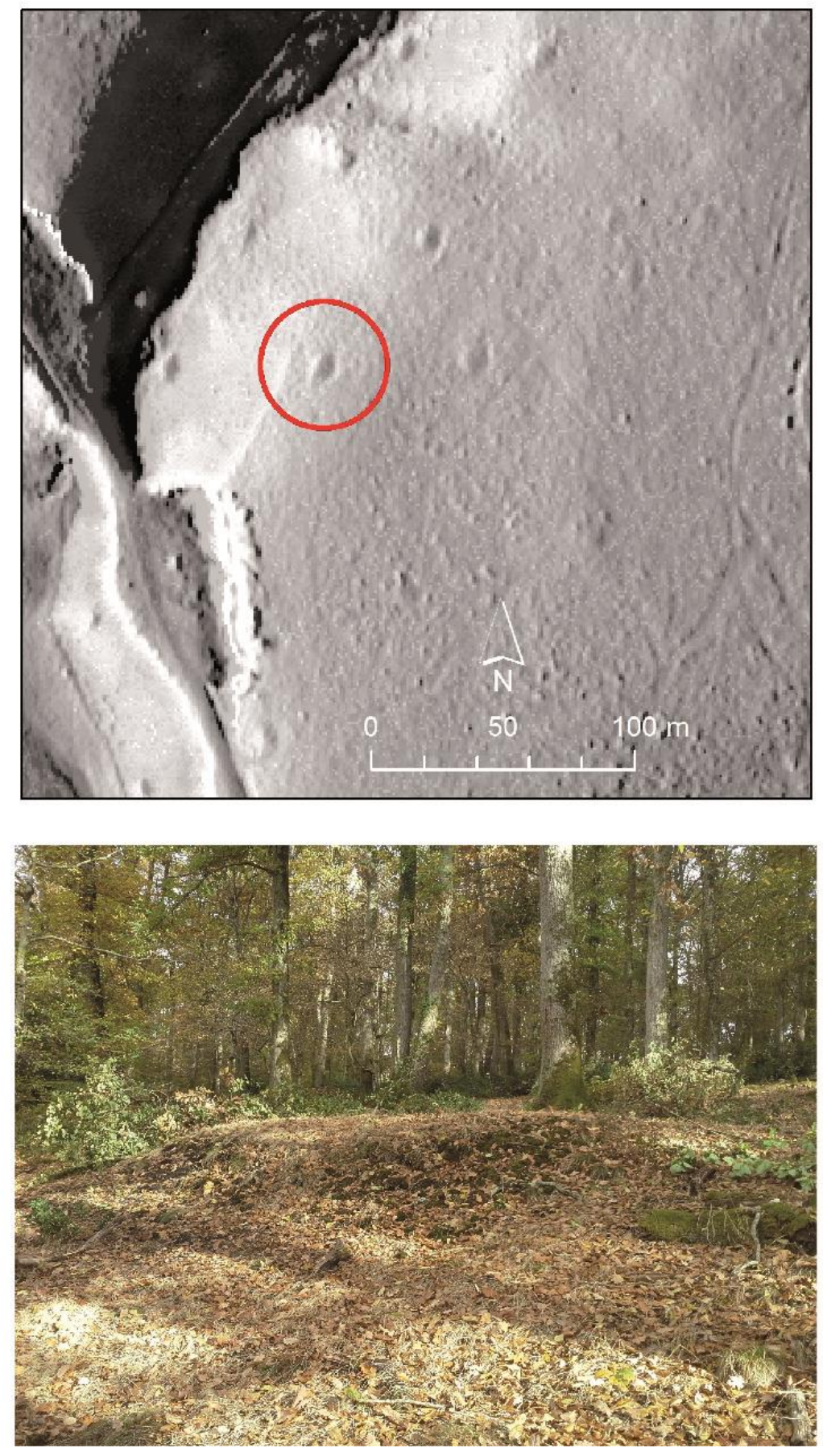

Figure 16. Exemple d'une charbonnière, telle qu'on la voit sur l'image LiDAR et sur le terrain (LiDAR : CRAIG-Fit Conseil 2016, cliché : L. Laüt)

\footnotetext{
${ }^{9}$ Au sujet de l'archéologie du charbonnage, voir entre autres, Bonhôte, Vernet 1988, Métalié (dir.) 1992, Acovitsioti-Hameau, A. 2001 ou encore Dupin et al. 2017.
} 
Notons que ces charbonnières sont aussi visibles dans les champs labourés, où les clichés aériens permettent d'observer des semis réguliers de taches sombres ${ }^{10}$. Mais alors, la charrue nivelle les reliefs et les images LiDAR ne permettent pas d'en retrouver la trace (fig. 17). Il faut donc jouer sur des approches complémentaires pour saisir l'étendue de ces activités passées.
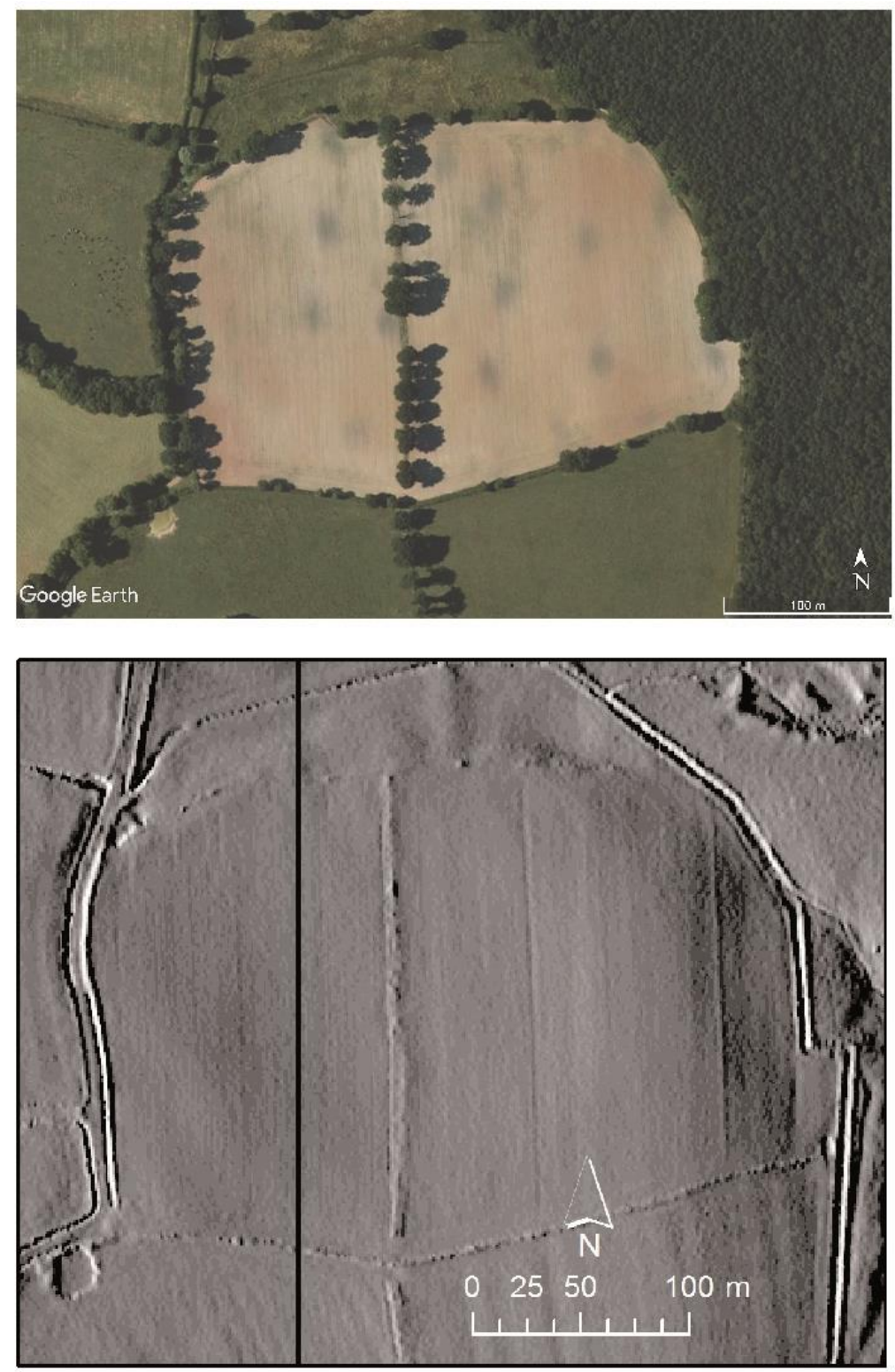

Figure 17. Exemple de charbonnières dans un champ en lisière de forêt actuelle, visibles en vue aérienne, mais invisibles sur l'image LiDAR (en haut : Google Earth, en bas : CRAIG-Fit Conseil 2016)

\subsection{Ce qu'on ne voit toujours pas}

Les structures de toutes époques en matériaux périssables échappent toujours à notre connaissance. Si la période romaine est bien représentée, avec ses constructions en dur, il faut avoir conscience de la sous-représentation des occupations pré et protohistoriques, mais aussi médiévales et modernes, ce qui est le lot commun des cartes archéologiques en milieu forestier ${ }^{11}$. Dans le secteur de Tronçais, il nous manque donc à peu près tous les bâtiments en terre et bois, des fermes gauloises aux loges de

\footnotetext{
10 Nous les avions également observées en Berry, à l'occasion des prospections menées autour d'Argentomagus (Dumasy, Dieudonné-Glad, Laüt 2010 : 73).

${ }^{11}$ Dans une étude menée pour l'INRA à l'échelle de la France, on peut constater que 68,5\% des sites archéologiques datés, répertoriés en milieu forestier, relèvent de l'Antiquité (Maussion 2003 : fig. 5).
} 
bucherons ou de charbonnier plus récentes ${ }^{12}$, même si ces dernières sont visibles sur les images LiDAR dans d'autres massifs, comme ceux de Chambord ${ }^{13}$ ou d'Orléans ${ }^{14}$. Ajoutons que même pour la période romaine, il nous manque une partie des structures, puisque d'une part les constructions en terre et bois sont également courantes à cette époque, d'autre part seule une partie des constructions en pierre apparait en relief. Ainsi, sur le site des Petits Jardins à Isle-et-Bardais, les murs de clôture du sanctuaire, non perceptibles en surface, n'ont été révélés que par les prospections géophysiques et la fouille (Laüt 2012).

En outre, aucun site funéraire n'a encore été formellement identifié dans ce secteur et nous ne pouvons faire que des hypothèses concernant quelques tertres observés par endroits. Mais l'acquisition LiDAR aura au moins permis de confirmer qu'il n'y a rien ici de comparable aux grandes nécropoles tumulaires qui sont conservés sous d'autres massifs, par exemple en forêt de Haguenau (Abert et al. 2010) ou d'Orléans (Dardignac et David 2018, Riou 2019).

\section{Conclusion}

Avec le développement de l'archéologie préventive et de ses grands décapages en rase campagne, la notion même de site archéologique a évolué, nous obligeant à considérer non pas quelques points ou lignes sur une carte, mais bien un tissu souvent ininterrompu de traces d'occupations anciennes. Le milieu forestier est quant à lui peu touché par de telles opérations, mais les images LiDAR y provoquent le même type de révolution. Les paysages anciens, fossilisés sous la forêt, ont encore des reliefs que les travaux agricoles ont estompés ailleurs, et l'emplacement des structures peut donc se lire à la surface du sol.

En forêt de Tronçais, la masse des informations livrées par les données LiDAR a considérablement complété et renouvelé notre vision archéologique du secteur. Les modèles numériques de terrain suffisent parfois pour identifier certains types d'aménagements : voies et chemins ou structures agraires, notamment. Mais les vérifications sur le terrain restent indispensables pour bien d'autres reliefs, afin d'exclure ce qui ne relève pas de l'archéologie, mais aussi de caractériser et dater les implantations humaines.

Sur les 774 indices anthropiques contrôlés depuis 2017, seulement 118 , soit 13,5\% correspondent à des sites archéologiques qui étaient déjà connus (fig. 18). Si la progression est notable pour les occupations antiques, médiévales ou modernes, ce sont aussi de très nombreuses structures encore non datées qui sont ainsi révélées et qu'il nous faudra tenter de caler chronologiquement ${ }^{15}$. Mais bon nombre d'entre elles (voies, terrasses, talus, fossés, tas d'épierrement, etc.) peuvent présenter un caractère assez largement pérenne, restant en usage sur la longue durée même si leurs fonctions, elles, ont pu évoluer. Le recours à la fouille et aux datations OSL peut s'avérer alors indispensable pour dénouer cet écheveau complexe, comme cela fut fait dans le cadre du programme SOLiDAR en région Centre (Laplaige et al. $2017: 53-63$ ).

\footnotetext{
${ }^{12}$ Citons à ce propos un registre manuscrit intitulé «Renseignements sur les loges des bucherons du département », daté de 1806, qui recense entre autres le nom, l'emplacement, et les occupants de chaque loge, dont 74 se trouvent à l'intérieur de la forêt de Tronçais (Archives départementales de l'Allier, réf. 7 M 5504).

${ }^{13}$ Laplaige et al. 2015 : 54-55 (86 loges recensées) et recherche doctorale en cours d'Aude Crozet à l'université de Tours.

14 Riou 2019 : 69-71 (42 loges recensées), Riou à paraître et recherche doctorale en cours à l'université Paris 1 PanthéonSorbonne.

${ }^{15}$ Les carottages pratiqués en avril 2019 en forêt de Tronçais par Christophe Petit, Damien Parrondo et Alain Giosa devraient nous apporter d'utiles précisions sur l'évolution du paysage dans ce secteur.
} 


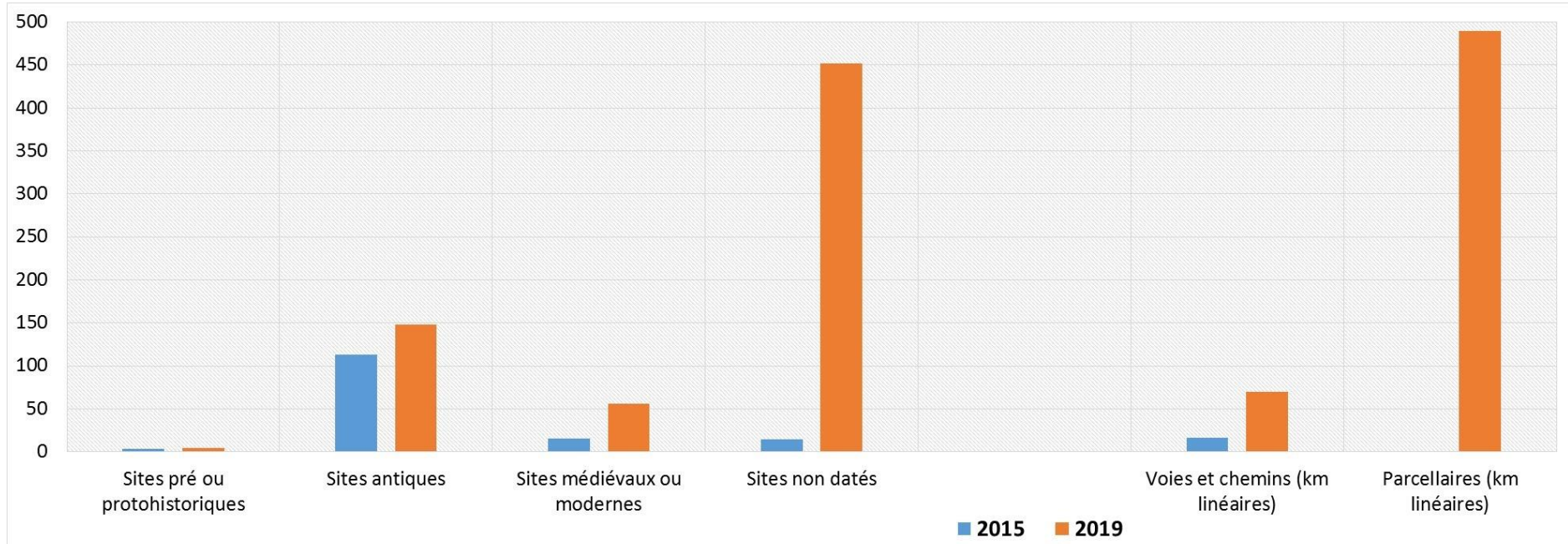

Figure 18. Comparaison des données de la carte archéologique, avant et après l'acquisition LiDAR de 2016 (L. Laüt)

La situation n'a en revanche guère évolué pour les structures pré ou protohistoriques, qui n'ont pas imprimé ici leur trace en relief à la surface du sol. Certaines formes d'occupations anciennes et certaines périodes continuent donc à être totalement absentes ou largement sous-représentées. À ce propos, signalons que 45 des 145 sites connus avant l'acquisition LiDAR, soit près d'un tiers, ne présentent aucun relief visible sur les modèles numériques de terrain ${ }^{16}$. Nous avons aussi pu constater que des charbonnières, pourtant observées sur le terrain, n'étaient pas détectées par la LiDAR, en raison de leur relief quasi inexistant ou de la densité de points plus faible par endroits. Il est important de garder ces réserves à l'esprit en interprétant les données disponibles, pour nombreuses et impressionnantes qu'elles soient.

\section{Bibliographie}

Abert, F., D. Bevilacqua, F. Delrieu et P. Rieth P. 2010. «Les tertres funéraires du Schirrheinerweg et du Kurzgeländ de la forêt de Haguenau », Revue d'Alsace, $136:$ 7-32.

Acovitsioti-Hameau, A. 2001. "Aller au charbon... Les sites de charbonnage et leur impact sur les paysages forestiers de la colline varoise (Provence, France) », Forêt Méditerranéenne, volume 22, 4 : 329-344.

Auclair, A. et D. Woronoff, 2005. Les forges de Tronçais : une usine au cæur de la forêt, Saint-Bonnet-Tronçais, CPIE Tronçais.

Bergès, L. et Dupouey, J.-L. 2017, «Écologie historique et ancienneté de l'état boisé : concepts, avancées et perspectives de la recherche », Revue Forestière Française, 69, 4 : 297-318.

Bertrand, É. 1980. «Les sites antiques en Pays de Tronçais, Inventaire sommaire », Bulletin des amis de la forêt de Tronçais, $25: 21-31$.

Bertrand, É. 1983 : «Les sites antiques en Pays de Tronçais, Inventaire complémentaire », Bulletin des amis de la forêt de Tronçais, $28: 37-40$.

Bertrand, É. 2000. «Le Pays de Tronçais, les sites antiques», La Lettre du Pays de Tronçais, 49, cartes à l'échelle 1/10000 et commentaires, 41 feuilles A3, 2000.

Bessard, A. 2018. Tronçais et Civrais dans les deux Guerres mondiales, Issoudun, Alice Lyner éditions.

Bonfils, P. 1970. «La forêt de Tronçais - Étude pédologique», Revue Forestière Française, 22 : 35-43.

Bonhôte, J. et J.-L. Vernet 1988. «La mémoire des charbonnières. Essai de reconstitution des milieux forestiers dans une vallée marquée par la métallurgie (Aston, Haute-Ariège) », Revue française forestière, XL.3 : 198-211.

\footnotetext{
16 Il s'agit principalement de sites repérés par les érudits locaux entre les années 1960 et 1990, qui se signalent par la seule présence de mobilier en surface (fragments de tuiles, de céramique, etc.).
} 
Bourdet, J.-R. 2000. «Analyse spatiale des relations entre utilisation ancienne du sol et caractéristiques du milieu forestier », mémoire de fin d'études pour l'obtention du titre d'ingénieur des techniques agricoles, INRA-Nancy.

Caraire, G. 2008. «Prospections géophysiques sur le site gallo-romain des Petits Jardins à Isle-et-Bardais (forêt domaniale de Tronçais, Allier)», mémoire de master 2 professionnel, université de Bordeaux 3.

Chevalier, J. 1940. La Forêt de Tronçais en Bourbonnais... $2^{e}$ édition augmentée, Paris, Éditions de la Chronique des lettres françaises.

Dambrine, E., J.-L. Dupouey, L. Laüt, L. Humbert, M. Thinon, T. Beaufils et H. Richard 2007. "Present Forest Biodiversity Patterns in France Related to Former Roman Agriculture", Ecology, 88, 6: 1430-1439.

Dardignac, C. et S. David 2018. «Rapport d'analyses et d'interprétation de données LiDAR - Forêt domaniale d'Orléans - Massif des Bordes (Loiret) », Fontainebleau, Office National des Forêts.

David, S. 2010. «L'occupation antique et le parcellaire fossile de la forêt de Sénart», mémoire de master 1, université Paris 1 Panthéon-Sorbonne.

David, S. 2011. «Étude de l'occupation du sol durant l'Antiquité et analyse des mares dans la forêt domaniale de Sénart et la forêt régionale de Rougeau. Essonne (91) et Seine-et-Marne (77)», mémoire de master 2, université Paris 1 Panthéon-Sorbonne.

Delaunay, A., J. P. Nebout, et T. Beaufils. 1991. «Bocage Bourbonnais - Forêt de Tronçais, Typologie des stations forestières », rapport scientifique de l'ONF, CRPF Auvergne, CEF Allier.

Diedhiou, A. G., J.-L. Dupouey, M. Buée, E. Dambrine, L. Laüt et J. Garbaye 2009. "Response of ectomycorrhizal communities to past Roman occupation in an oak forest", Soil Biology and Biochemistry, 41, 10 : 2206-2213.

Diedhiou, A. G., J.-L. Dupouey, M. Buée, E. Dambrine, L. Laüt et J. Garbaye 2010. "The functional structure of ectomycorrhizal communities in an oak forest in central France witnesses ancient Gallo-Roman farming practices", Soil Biology and Biochemistry, 42, 5: 860-862.

Dumasy, F., N. Dieudonné-Glad et L. Laüt L. 2010. Travail de la terre, travail du fer: l'espace rural autour d'Argentomagus, Saint-Marcel, Indre, Mémoires, 23, Bordeaux, Ausonius.

Dupin, A., O. Girardclos, C. Fruchart, C. Laplaige, L. Nuninger, A. Dufraisse, E. Gauthier 2017. "Anthracology of charcoal kilns in the forest of Chailluz (France) as a tool to understand Franche-Comte forestry from the mid- $15^{\text {th }}$ to the early $20^{\text {th }}$ century AD", Quaternary International, $458: 200-213$.

Fernandez-Diaz J. C., W. E. Carter, R. L. Shrestha, C. L. Glennie 2014. "Now You See It... Now You Don't: Understanding Airborne Mapping LiDAR Collection and Data Product Generation for Archaeological Research in Mesoamerica", Remote Sensing, 6, 10 : 9951-10001.

Fleury, J. et A. 1665. Forest de Tronsais... Document cartographique manuscrit au 1/8000 environ, 30 feuilles vélin assemblées (3040 x 2340 m), Bibliothèque nationale, département des manuscrits.

Gandini, C., F. Dumasy et L. Laüt 2013. «Paysages économiques du territoire des Bituriges Cubes, approche comparée de trois modes d'occupation du sol», in: J.-L. Fiches, R. Plana-Mallart et V. Revilla Calvo (dir.) 2013. Actes du IX colloque AGER de Barcelone (25-27 mars 2010), Montpellier, Presses universitaires de Méditerranée : 67-81.

Georges-Leroy, M. 2009. «Le massif forestier, objet pertinent pour la recherche archéologique. L'exemple du massif forestier de Haye (Meurthe-et-Moselle)», Revue géographique de l'Est, 49 (2-3) : 1-16.

Georges-Leroy, M. 2011. "Apport du LiDAR à la connaissance de l'histoire de l'occupation du sol en forêt de Haye », ArchéoSciences - revue d'archéométrie, 35 : 117-129.

Giosa, A. 2011. «Les marqueurs de l'activité anthropique en forêt de Tronçais, étude du bassin-versant de Saint-BonnetTronçais (Allier). Une approche géoarchéologique en milieu forestier », mémoire de master 1, université Paris 1 Panthéon-Sorbonne.

Humbert, L. 2002. «Relation entre l'utilisation du sol à l'époque gallo-romaine et la biodiversité actuelle en forêt de Tronçais », rapport de stage, maîtrise de Biologie des populations et des écosystèmes, INRA-Nancy.

Laplaige, C., X. Rodier, L. Magiorani et A. Corzet 2015. «SOLiDAR : Diachronie de l'occupation du sol : télédétection LiDAR en forêt de Chambord, Boulogne, Russy, Blois », Rapport d'opération, université François Rabelais, Tours.

Laplaige, C, K. Coulibaly, A. Crozet, P. Gardère, A. Lacoste et al. 2017. SOLiDAR : Diachronie de l'occupation du sol : télédétection LiDAR en forêts de Chambord, Boulogne, Russy et Blois, Rapport de recherche, UMR 7324 CITERES Laboratoire Archéologie et Territoires. 
Laüt, L. 2004. «Le paysage antique de la forêt de Tronçais, bilan des travaux d'Elie Bertrand et des recherches récentes », Bulletin de la société des amis de la forêt de Tronçais, 49 : 49-86.

Laüt, L. 2007. "Caractérisation des sites antiques dans les forêts du Berry et du Bourbonnais", in: J.-L. Dupouey, Dambrine E., Dardignac C. et Geroges-Leroy M. (dir.), La mémoire des forêts, actes du colloque "Archéologie, forêt et environnement» (Sylva 2004), 14-16 décembre 2004, ONF, INRA, DRAC Lorraine, Velaine-en Haye : 77-85.

Laüt, L. 2011. «Le sanctuaire des Petits Jardins à Isle-et-Bardais, en forêt domaniale de Tronçais : résultats des fouilles menées depuis $2002 »$, Bulletin de la société des amis de la forêt de Tronçais, 56 : 37-61.

Laüt, L. 2012. "Premier bilan des recherches sur le sanctuaire des Petits Jardins à Isle-et-Bardais, en forêt domaniale de Tronçais (Allier)", in: O. de Cazanove et Méniel P. (dir.), Étudier les lieux de culte de la Gaule romaine, Montagnac, Monique Mergoil : 181-196.

Laüt, L. 2013a. «Des monuments en pleine campagne : Marqueurs du paysage ? Marqueurs de la mémoire? À propos du sanctuaire antique d'Isle-et-Bardais (Allier) et de quelques autres sites de Gaule centrale », in : Monumentalités et mémoire, Actes de la $2^{e}$ Journée d'étude sur le thème de la monumentalité, 30 novembre 2012, INHA, publication en ligne de l'Hicsa, Paris (https://hicsa.univ-paris1.fr).

Laüt, L. 2013b. «Le site des Petits Jardins dans la forêt de Tronçais (Isle-et-Bardais, Allier), données récentes sur le sanctuaire antique et l'atelier de tuilier du haut Moyen Age», in : Bilan d'activité 2012 de la DRAC Auvergne, Journée régionale de l'archéologie, Clermont-Ferrand 2013, Ministère de la culture et de la communication, Clermont-Ferrand, DRAC-Auvergne : 112-113.

Laüt, L. 2015. «Bilan de la campagne de fouille 2014 sur le site des Petits Jardins à Isle-et-Bardais, en forêt domaniale de Tronçais », Bulletin de la Société des Amis de la Forêt de Tronçais, 60 : 52-60.

Laüt, L. 2018. «L'exploitation archéologique des données LiDAR sur la forêt de Tronçais : un premier bilan », Bulletin de la Société des Amis de la forêt de Tronçais, 62 : 43-59.

Laüt, L. et M. Guillon M 2016. «Opération LIDAR en forêt de Tronçais : du projet à la réalisation », Bulletin de la Société des Amis de la forêt de Tronçais, 61 : 24-34.

Laüt, L., J.-L. Dupouey, É. Dambrine et L. Humbert 2014. «La forêt domaniale de Tronçais (Allier), approche archéologique et environnementale de l'occupation antique », in : Sylva et saltus en Gaule romaine, actes du VII ${ }^{c}$ colloque AGER de Rennes, 27-28 octobre 2004, Environnement, société et archéologie (19), Besançon, Annales littéraires de l'université de Franche-Comté : 213-226.

Lulin, J.-M. 1979. «Géologie de la forêt de Tronçais », Bulletin des Amis de la forêt de Tronçais, 24 : 5-16.

Maussion, A. 2003. "Occupation ancienne du sol et milieux forestiers actuels en France métropolitaine, synthèse bibliographique », rapport d'étude, Champenoux, INRA-Nancy.

Métalié, J.P. (dir.) 1992. Protoindustries et histoire de forêts, Actes du colloque de Loubières, Ariège, octobre 1990, Les Cahiers de l'ISARD, 3.

Mollard, J. 2017. «Acquisition LiDAR en forêt de Tronçais : premier résultat sur la donnée forestière », Bulletin de la Société des amis de la forêt de Tronçais, 62 : 17-26.

Piboule, M. et É. Bertrand 1995. Mémoire des communes bourbonnaises, Au pays de la forêt, Au long des vieux chemins de Tronçais, Montluçon, Foyers ruraux de l'Allier.

Raffignon, G. et J. Chevalier 1913. La Forêt de Tronçais, notice descriptive et historique, Limoges, Decourtieux et Gout.

Riou, A. 2019. «Les paysages anciens de la forêt d'Orléans : l'exploitation des données LiDAR du massif de Lorris-les Bordes (Loiret)», mémoire de master 2 en archéologie, université Paris 1 Panthéon-Sorbonne.

Riou, A. à paraître : «L'exploitation archéologique des données LiDAR du massif de Lorris-Les Bordes (Loiret) », Revue archéologique du Loiret, 2019, 40.

Romane, J. et P. 2001. Fontaines et sources de la forêt de Tronçais, Bourbon-l'Archambault, Éd. l'Échoppe.

Trier, Ø. D. and L. Holger Pilø 2015. "Archaeological Mapping of Large Forested Areas, Using Semi-Automatic Detection and Visual Interpretation of High-Resolution LiDAR Data" in Giligny, F., F. Djindjian, L. Costa, P. Moscati and S. Robert (ed.), CAA2014 21 ${ }^{\text {st }}$ Century Archaeology Concepts, methods and tools, Proceedings of the $42^{\text {nd }}$ Annual Conference on Computer Applications and Quantitative Methods in Archaeology, Archaeopress, Oxford: 81-86. 